\title{
Navier-Stokes Equations on Lipschitz Domains in Riemannian Manifolds
}

\author{
Marius Mitrea* and Michael Taylor ${ }^{\dagger}$
}

\section{Introduction}

The Navier-Stokes equations are a system of nonlinear evolution equations modeling the flow of a viscous, incompressible fluid. One ingredient in the analysis of this system is the stationary, linear system known as the Stokes system, a boundary value problem (BVP) that will be described in detail in the next section.

Layer potential methods in smoothly bounded domains in Euclidean space have proven useful in the analysis of the Stokes system, starting with work of Odqvist and Lichtenstein, and including work of Solonnikov and many others. See the discussion in Chapter III of [10] and in [17], for the case of flow in regions with smooth boundary. A treatment based on the modern language of pseudodifferential operators can be found in [18].

In 1988, E. Fabes, C. Kenig and G. Verchota [6], extended this classical layer potential approach to cover Lipschitz domains in Euclidean space. In [6] the main result concerning the (constant coefficient) Stokes system on Lipschitz domains with connected boundary in Euclidean space, is the treatment of the $L^{2}$-Dirichlet boundary value problem (and its regular version). To achieve this, the authors solve certain auxiliary Neumann type problems and then exploit the duality between these and the original BVP's at the level of boundary integral operators. P. Deuring and W. von Wahl [4] made use of the analysis in [6] to demonstrate the short-time existence of solutions to the Navier-Stokes equations in bounded Lipschitz domains in threedimensional Euclidean space. It was necessary in [4] to include the hypothesis that the boundary be connected.

The hypothesis that the boundary be connected pervaded much work on the application of layer potentials to analysis on Lipschitz domains. It was certainly natural to speculate that this restriction was an artifact of the methods used and not

\footnotetext{
*Partly supported by NSF grant DMS-9870018.

†Partially supported by NSF grant DMS-9877077. 1991 Mathematics Subject Classification. Primary 35Q30, 76D05, 35J25; Secondary 42B20, 45E05. Key words: Navier-Stokes equations, Lipschitz domains, layer potentials, boundary problems.
} 
a necessary condition. Looking into this matter has provided us the impetus for a program that has produced [12], [13], and [11]. One thing we learned was that the decision to extend the study from constant-coefficient PDE to equations with variable coefficients tends to erase such a topological obstruction. Making this decision, we are then led to study a variety of boundary problems for the Laplace operator and other elliptic operators on Lipschitz domains in Riemannian manifolds. Carrying out this extension requires further work, but this has led to many results on Dirichlet, Neumann, and other natural boundary problems for the Laplace operator and the Hodge Laplacian in [12], [13], and [11].

Here we take up the study of the Stokes system in such a framework. In the first part of this paper we refine and extend the work in [6] in several important respects, analyzing the Stokes system on arbitrary Lipschitz domains on a compact Riemannian manifold. Whereas in our previous works we have allowed metric tensors of rather limited smoothness, in this work we assume the metric tensor is smooth, merely to moderate the level of technicalities.

At the core of our program is the study of the layer potential operators naturally associated with the PDE under discussion. In order to carry out this segment of analysis we need to further polish some of the results in [11], [12] and [13]. As in [6] we make essential use of a Rellich type identity but, unlike [6], our approach is based entirely on the use of a single layer potential (both for the plain $L^{2}$-Dirichlet problem as well as for its more regular versions). Again the flexibility to work on manifolds with nontrivial curvature is crucial in allowing us to dispense with any artificial topological hypotheses on the underlying domain.

In a broad outline, our strategy involves four main steps: (1) constructing a global fundamental solution and introducing the corresponding layer potentials, (2) inverting the single layer potential (via a parametrix) on the boundary of smooth domains, (3) deriving a priori estimates uniform with respect to the Lipschitz character of the domain, and (4) solving the problem in arbitrary Lipschitz domains via layer potentials.

Using this method we produce global integral representation formulas for the solution. In turn, these can be used to establish some very useful global regularity results in Sobolev-Besov scales. For example, here we use these to describe the smoothness of elements in the domain of the Stokes operator $A$ in a Lipschitz domain $\Omega$. One particular example is the inclusion

$$
\operatorname{Dom}(A) \hookrightarrow \bigcap_{|p-2|<\varepsilon} B_{1+1 / p}^{p, p^{*}}(\Omega) \text { for some } \varepsilon=\varepsilon(\Omega)>0,
$$

where $p^{*}:=\max \{p, 2\}$. This type of result is the departure point in our analysis of the (non-linear, time-dependent) Navier-Stokes equations which we undertake in $\S 9$. Taking the Fujita-Kato functional analytic approach, we prove the existence of local strong solutions for the initial value problem for the Navier-Stokes equations in Lipschitz cylinders in dimension $\leq 3$. 
The layout of the paper is as follows. In $\S 2$ we introduce some basic notation and discuss the general set-up for the stationary Stokes system with nonhomogeneous boundary conditions. Section 3 contains a detailed treatment (with emphasis on jump-relations and mapping properties) of the layer potential operators naturally associated with the Stokes system. Next, in $\S 4$, we establish the relevant Rellich type estimate for solutions of the Stokes system, and in $\S 5$ we establish a useful pressure estimate. These estimates are then used in $\S 6$ to prove the invertibility of the single layer potential operator (cf. Theorem 6.1). Here we also make use of our earlier results for the Dirichlet problem for the Laplace-Beltrami operator on manifolds; cf. [12], [13]. Results on existence for the $L^{p}$-Dirichlet BVP, as well as naturally accompanying regularity results, in arbitrary Lipschitz domains, are stated and proved in $\S 7$ for $p$ near 2 ; see Theorem 7.1. The Stokes operator is introduced in $\S 8$ and the inclusion (1.1) is proved there. Finally, in $\S 9$, we tackle the initial value problem for the Navier-Stokes equations.

\section{The Stokes system}

Let $M$ be a smooth, compact, boundaryless manifold of (real) dimension $m$. As usual, by $T M$ and $T^{*} M$ we denote, respectively, the tangent and cotangent bundle on $M$. Also, $\Lambda^{\ell} T M$ stands for the corresponding (exterior) power bundle.

Next, if $M$ is equipped with a smooth Riemannian metric tensor $g=\sum_{j, k} g_{j k} d x_{j} \otimes$ $d x_{k}$, denote by $\left(g^{j k}\right)_{j k}$ the inverse matrix to $\left(g_{j k}\right)$ and set $g:=\operatorname{det}\left(g_{j k}\right)_{j k}$. Recall that $\operatorname{div} X:=g^{-1 / 2} \partial_{j}\left(g^{1 / 2} X_{j}\right)$ if $X=X_{j} \partial / \partial x_{j}$ and $\operatorname{grad} f=g^{j k}\left(\partial f / \partial x_{j}\right) \partial / \partial x_{k}$ are the usual divergence and gradient operators.

The pairing $\left\langle d x_{j}, d x_{k}\right\rangle:=g^{j k}$ defines an inner product in $\Lambda^{1} T M$. With respect to this, grad and - div are adjoint to each other. More generally, abbreviate $d x_{i_{1}} \wedge$ $d x_{i_{2}} \wedge \ldots \wedge d x_{i_{\ell}}$ by $d x_{I}$, where $I=\left(i_{1}, i_{2}, \ldots, i_{\ell}\right)$ and wedge stands for the ordinary exterior product of forms. Then, $\left\langle d x_{I}, d x_{J}\right\rangle:=\operatorname{det}\left(\left(g^{i j}\right)_{\substack{i \in I \\ j \in J}}\right),|I|=|J|=\ell$, defines an inner product in $\Lambda^{\ell} T M$ for each $1 \leq \ell \leq m$. If, as usual, we let $d$ and $\delta$ stand, respectively, for the exterior derivative and exterior co-derivative operators, then $d$ and $\delta$ are adjoint to each other. The Hodge Laplacian is then given by

$$
\Delta:=-(d \delta+\delta d)
$$

As is customary, we may identify vector fields with one-forms (i.e., $T M \cong T^{*} M=$ $\left.\Lambda^{1} T M\right)$ via $\partial / \partial x_{j} \mapsto g_{j k} d x_{k}$ (lowering indices). This mapping is an isometry whose inverse (raising indices) is given by $d x_{j} \mapsto g^{j k} \partial / \partial x_{k}$. Under this identification, $\operatorname{grad}: C^{\infty}(M) \rightarrow C^{\infty}(M, T M)$ becomes $d: C^{\infty}(M) \rightarrow C^{\infty}\left(M, \Lambda^{1} T M\right)$ and div : $C^{\infty}(M, T M) \rightarrow C^{\infty}(M)$ becomes $-\delta: C^{\infty}\left(M, \Lambda^{1} T M\right) \rightarrow C^{\infty}(M)$.

Furthermore, if $\nabla$ is the Levi-Civita connection and Ric is the Ricci tensor on $M$ then, under the above identification, the Bochner Laplacian and the Hodge Laplacian are related by 


$$
-\nabla^{*} \nabla \equiv \Delta+\text { Ric }
$$

a special case of the Weitzenbock identity.

We let $O P S^{\ell}$ denote the collection of all classical pseudodifferential operators $P(x, D)$ of order $\ell$. In particular, their symbols $p(x, \xi) \in S_{1,0}^{\ell}$ satisfy $p(x, \xi) \sim$ $p_{\ell}(x, \xi)+p_{\ell-1}(x, \xi)+\cdots$, where $p_{j}(x, \xi)$ is smooth and homogeneous of degree $j$ in $\xi$ for $|\xi| \geq 1, j=\ell, \ell-1, \ldots$. Also, we denote by $\sigma(P ; x, \xi)$ the principal symbol $p_{\ell}(x, \xi)$.

For a vector field $X$, set $\mathcal{L}_{X}$ for the Lie derivative in the direction of $X$. In particular, the deformation tensor $\operatorname{Def} X$ is defined as $\frac{1}{2} \mathcal{L}_{X} g$, where $g$ stands for the metric tensor. It follows that $\operatorname{Def} X$ is a symmetric tensor field of type $(0,2)$ and

$$
(\operatorname{Def} X)(Y, Z)=\frac{1}{2}\left\{\left\langle\nabla_{Y} X, Z\right\rangle+\left\langle\nabla_{Z} X, Y\right\rangle\right\}, \quad \forall X, Y, Z \in T^{*} M .
$$

Thus, Def : $C^{\infty}(M, T M) \rightarrow C^{\infty}\left(M, S^{2} T^{*} M\right)$, where $S^{2} T^{*} M$ stands for symmetric tensor fields of type $(0,2)$. In coordinate notation,

$$
(\operatorname{Def} X)_{j k}=\frac{1}{2}\left(X_{j ; k}+X_{k ; j}\right), \quad \forall j, k .
$$

Here, as usual, for a vector field $X=\sum_{j} X^{j} \partial_{j}$, we set $X_{k ; j}:=\partial_{j} X_{k}-\sum_{l} \Gamma_{k j}^{l} X_{l}$, where $X_{k}=\sum_{l} g_{k l} X^{l}$ and $\Gamma_{k j}^{l}$ are the Christoffel symbols associated with the metric.

If $\widetilde{u} \in T^{*} M \equiv \Lambda^{1} T M$ corresponds to $u \in T M$ via the metric tensor, then the principal symbol of the deformation operator is

$$
-i \sigma(\operatorname{Def} ; x, \xi) u=\frac{1}{2}(\xi \otimes \widetilde{u}+\widetilde{u} \otimes \xi)=: \xi \otimes_{S} \widetilde{u} .
$$

at each $x \in M$ and for each $\xi \in T_{x}^{*} M, u \in T_{x} M$. The adjoint of Def is $\operatorname{Def}^{*} v=-\operatorname{div} v$, $v \in S^{2} T^{*} M$, or in local coordinates, $\left(\operatorname{Def}^{*} v\right)^{j}=-v_{; k}^{j k}$. For further reference, let us also point out that at each $x \in M$

$$
i \sigma\left(\text { Def }^{*} ; x, \xi\right)\left(u \otimes_{S} v\right)=\frac{1}{2}(\langle u, \xi\rangle v+\langle v, \xi\rangle u), \quad \forall \xi \in T_{x}^{*} M, \quad \forall u, v \in T_{x} M
$$

In particular,

$$
\sigma\left(\operatorname{Def}^{*} \operatorname{Def} ; x, \xi\right)=\frac{1}{2}|\xi|^{2}\left(I+P_{\xi}\right)
$$

where $P_{\xi}(u):=|\xi|^{-2}\langle\xi, u\rangle \xi$ is the projection of $u$ along $\xi$ (identified as a vector).

Consider an arbitrary Lipschitz subdomain $\Omega$ of $M$. This means that, in local coordinates, $\partial \Omega$ can be described in terms of graphs of Lipschitz functions. We are interested in the (stationary) linearized Navier-Stokes equations equipped with a 
nonhomogeneous boundary condition:

$$
(B V P)\left\{\begin{array}{l}
u \in C_{\mathrm{loc}}^{2}\left(\Omega, \Lambda^{1} T M\right), p \in C_{\mathrm{loc}}^{1}(\Omega), \\
L u+d p=0 \text { in } \Omega, \\
\delta u=0 \text { in } \Omega \\
\left.u\right|_{\partial \Omega}=f \text { on } \partial \Omega .
\end{array}\right.
$$

Here the second-order partial differential operator $L$ is given by

$$
L:=2 \text { Def }^{*} \text { Def }=\nabla^{*} \nabla-\operatorname{grad} \operatorname{div}-\operatorname{Ric} \equiv-\Delta+d \delta-2 \text { Ric. }
$$

Note that on divergence-free forms $u$, the action of the operator $L$ simplifies to $L u=$ $-\Delta u-2$ Ric $u$. In the particular case when $\operatorname{Ric}=-\lambda I$ on $M$, this further reduces to $L u=(2 \lambda-\Delta) u$. As noted in [5], the natural operator for the Stokes system on an arbitrary Riemannian manifold is (2.8). See also the treatment in [18].

In the sequel, we shall not make any notational distinction between a vector field and its associated 1-form (i.e., we shall tacitly identify $T^{*} M \equiv \Lambda^{1} T M$, as has been the case in the last leg of (2.8)). Also, we shall occasionally drop the dependence of the various spaces of forms on the exterior power bundle.

\section{$3 \quad$ Layer potential operators}

Let $\Omega \subset M$ be a connected Lipschitz domain in a smooth, compact manifold $M$. Altering $M$ at will off $\bar{\Omega}$, matters can be arranged so that:

$$
M \text { has no nontrivial Killing fields, }
$$

and

$$
\Omega_{-}=M \backslash \bar{\Omega} \text { is connected. }
$$

Note that we can ensure (3.2) even when $\partial \Omega$ is not connected. That (3.1) can be arranged is "well known" to differential geometers. We mention a short proof. Picking $p \in \Omega_{-}$, we can arrange that the scalar curvature of our metric tensor have a unique, nondegenerate maximum at $p$, so all isometries of $M$ must fix $p$. Furthermore (if dim $M \geq 3$ ) for a generic such metric the Ricci tensor at $p$ will have distinct eigenvalues;

this can be seen by looking as the surface $y=\sum a_{j} x_{j}^{2}$; at the tip $x=0$ the eigenvalues of Ric are of the form $2 a_{j}\left(A-a_{j}\right)$, where $A=\sum a_{j}$, and generically these are all distinct in dimension $\geq 3$. In dimension 2 one can see that the circles $\{x: \operatorname{dist}(x, p)=$ $\varepsilon\}$ generically do not have constant Gauss curvature. In either case there cannot be any nontrivial one-parameter groups of isometries.

Now (3.1) guarantees that Ker Def $=\{0\}$. In particular, the elliptic operator 


$$
L:=2 \operatorname{Def}^{*} \operatorname{Def}: L_{1}^{2}(M, T M) \rightarrow L_{-1}^{2}(M, T M)
$$

is invertible. We seek

$$
\Phi \in O P S^{-2}\left(M ; \Lambda^{1} T M, \Lambda^{1} T M\right), \quad \Psi \in O P S^{-1}\left(M ; \Lambda^{1} T M, \mathbb{R}\right),
$$

so that

$$
L \Phi+d \Psi=I \text { and } \delta \Phi=0 \text { on } \mathcal{D}^{\prime}\left(M ; \Lambda^{1} T M\right) .
$$

Bring in the Hodge decomposition of 1-forms on $M$ :

$$
I=d \delta G+\delta d G+P_{h}=P_{d}+P_{\delta}+P_{h}=P_{d}+P_{d}^{\perp},
$$

where $P_{h}$ is the orthogonal projection onto $\mathcal{H}(M)$, the (finite dimensional) space of all harmonic forms on $M$, and $G$ is the Green operator at the level of 1-forms (i.e., $G=\Delta^{-1} P_{h}^{\perp}$ where $\Delta^{-1}$ is the inverse of $\Delta$ on $\left.\mathcal{H}(M)^{\perp}\right)$. Also, $P_{d}:=d \delta G, P_{\delta}:=\delta d G$ are projections onto the ranges of $d$ and $\delta$, respectively. Consider next

$$
\widetilde{L}:=P_{d}^{\perp} L P_{d}^{\perp}+P_{d} L P_{d} \in O P S^{2}(M ; T M, T M) .
$$

It is self-adjoint, elliptic and also invertible from $L_{1}^{2}(M, T M)$ onto $L_{-1}^{2}(M, T M)$. Set

$$
\Phi:=P_{d}^{\perp} \widetilde{L}^{-1} \in O P S^{-2}\left(M ; \Lambda^{1} T M, \mathbb{R}\right) .
$$

We have

$$
P_{d}^{\perp} L \Phi=P_{d}^{\perp} L P_{d}^{\perp} \Phi=\widetilde{L} \Phi=P_{d}^{\perp},
$$

since $P_{d}^{\perp}$ commutes with $\widetilde{L}^{-1}$, and $\delta \Phi=0$. Write (3.9) as $P_{d}^{\perp}(L \Phi-I)=0$, which gives

$$
L \Phi-I=\left(I-P_{d}^{\perp}\right)(L \Phi-I)=d \delta G(L \Phi-I) .
$$

Thus (3.5) holds if we set

$$
\Psi:=-\delta G(L \Phi-I) \in O P S^{-1}\left(M ; \Lambda^{1} T M, \Lambda^{1} T M\right) .
$$

Denote by $\Gamma(x, y)$ and $\Theta(x, y)$ the Schwartz kernels of $\Phi$ and $\Psi$, respectively. Then, on $M$,

$$
\left\{\begin{array}{l}
L_{x} \Gamma(x, y)+d_{x} \Theta(x, y)=\operatorname{Dirac}_{y}(x), \\
\delta_{x} \Gamma(x, y)=0,
\end{array}\right.
$$

where Dirac $y$ is the Dirac distribution with mass at $y$. It is worth pointing out that

$$
\Phi^{*}=\Phi \Longrightarrow \Gamma^{t}(x, y)=\Gamma(y, x) .
$$


At the level of principal symbols, the first observation is that

$$
\sigma(\Phi ; x, \xi) \text { is even in } \xi, \quad \sigma(\Psi ; x, \xi) \text { is odd in } \xi .
$$

Let us next take a closer look at the symbol $\sigma(\Phi ; x, \xi)$. Note that the identity $I=$ $\Delta G+P_{h}$ translates into $\sigma(G ; x, \xi)=|\xi|^{-2}, \forall \xi \in T_{x}^{*} M$, since $P_{h}$ is smoothing. Thus, $\sigma\left(P_{d} ; x, \xi\right)=|\xi|^{-2}(\xi \wedge(\xi \vee \cdot))$ and $\sigma\left(P_{d}^{\perp} ; x, \xi\right)=|\xi|^{-2}(\xi \vee(\xi \wedge \cdot))$. Accordingly, taking (2.7) into account,

$$
\sigma(\widetilde{L} ; x, \xi)=|\xi|^{2}+\xi \wedge(\xi \vee \cdot)=|\xi|^{2}\left(I+P_{\xi}\right),
$$

after some straightforward algebra (recall that $P_{\xi}$ is the orthogonal projection along $\xi)$. Hence,

$$
\begin{aligned}
\sigma(\Phi ; x, \xi) & =\sigma\left(P_{d}^{\perp} ; x, \xi\right) \sigma\left(\widetilde{L}^{-1} ; x, \xi\right)=|\xi|^{-4} \xi \vee\left(\xi \wedge\left(I+P_{\xi}\right)^{-1}\right) \\
& =|\xi|^{-4} \xi \vee\left(\xi \wedge\left(I-\frac{1}{2} P_{\xi}\right)\right)=|\xi|^{-4} \xi \vee(\xi \wedge \cdot)
\end{aligned}
$$

and, consequently,

$$
\langle\sigma(\Phi ; x, \xi) u, u\rangle=\frac{1}{|\xi|^{4}}|\xi \wedge u|^{2}=\frac{1}{|\xi|^{4}}\left[|u|^{2}|\xi|^{2}-|\xi \vee u|^{2}\right],
$$

by the Pythagorean theorem. In particular, for any $x \in \partial \Omega$ and $\xi \in T_{x}^{*} \partial \Omega$ (extended to a functional on $T_{x} M$ by setting $\langle\xi, \nu\rangle=0$ ) with $|\xi|=1$, we have

$$
\begin{aligned}
& \int_{-\infty}^{+\infty}\langle\sigma(\Phi ; x, \xi+t \nu) u, u\rangle d t \\
& \quad=\int_{-\infty}^{+\infty} \frac{1}{\left(1+t^{2}\right)^{2}}\left[\left(1+t^{2}\right)|u|^{2}-|\xi \vee u|^{2}-2 t\langle\xi \vee u, \nu \vee u\rangle-t^{2}|\nu \vee u|\right] d t \\
& \quad=\left(\int_{-\infty}^{+\infty} \frac{d t}{1+t^{2}}\right)|u|^{2}-\left(\int_{-\infty}^{+\infty} \frac{d t}{\left(1+t^{2}\right)^{2}}\right)|\xi \vee u|^{2}-\left(\int_{-\infty}^{+\infty} \frac{t^{2} d t}{\left(1+t^{2}\right)^{2}}\right)|\nu \vee u|^{2} \\
& \quad=\pi\left[|u|^{2}-\frac{1}{4}|\langle\xi, u\rangle|^{2}-\frac{3}{4}|\langle\nu, u\rangle|^{2}\right] \geq \frac{\pi}{4}|u|^{2}
\end{aligned}
$$

where the last step uses the obvious estimate $|u|^{2} \geq|\langle\xi, u\rangle|^{2}+|\langle\nu, u\rangle|^{2}$.

Fix now a Lipschitz domain $\Omega \subset M$ and denote by $\nu$ the outward unit conormal to $\partial \Omega$ and by $d \sigma$ the surface measure on $\partial \Omega$. Also, recall the usual scales of Sobolev and Besov spaces denoted here, respectively, by $L_{s}^{p}$ and $B_{s}^{p, q}$; cf. [1] and [8]. For $0 \leq s \leq 1$, we set

$$
L_{s, \nu}^{p}(\partial \Omega):=\left\{f \in L_{s}^{p}\left(\partial \Omega, \Lambda^{1} T M\right) ; \int_{\partial \Omega}\langle f, \nu\rangle d \sigma=0\right\}
$$


When $s=0$ we simply write $L_{\nu}^{p}(\partial \Omega)$.

Next, consider the single layer potential

$$
\mathcal{S} g(x):=\int_{\partial \Omega}\langle\Gamma(x, y), g(y)\rangle d \sigma(y), \quad x \in M \backslash \partial \Omega .
$$

Also, set

$$
S g(x):=\int_{\partial \Omega}\langle\Gamma(x, y), g(y)\rangle d \sigma(y), \quad x \in \partial \Omega
$$

and

$$
\mathcal{Q} g(x):=\int_{\partial \Omega}\langle\Theta(x, y), g(y)\rangle d \sigma(y), \quad x \in M \backslash \partial \Omega .
$$

Finally, we set $\Omega_{+}:=\Omega, \Omega_{-}:=M \backslash \bar{\Omega}$, and let $\left.\cdot\right|_{\partial \Omega_{ \pm}}$be the nontangential boundary trace operators on $\partial \Omega_{ \pm}$. That is,

$$
\left.u\right|_{\partial \Omega_{ \pm}}(x):=\lim _{y \in \gamma_{ \pm}(x)} u(y), \quad x \in \partial \Omega,
$$

where $\gamma_{ \pm}(x) \subseteq \Omega_{ \pm}$are appropriate nontangential approach regions. Finally, we let $(\cdot)^{*}$ stand for the nontangential maximal operator acting on $u$ defined in $\Omega_{+}$or $\Omega_{-}$ by

$$
u^{*}(x):=\sup \left\{|u(y)| ; y \in \gamma_{ \pm}(x)\right\}, \quad x \in \partial \Omega,
$$

(the choice of the sign \pm depends on where $u$ is defined). See, e.g., [20], [12] for more details.

Theorem 3.1 Let $\Omega$ be a Lipschitz domain. Then the following hold.

(1) For $1<p<\infty$,

$$
\left\|(\nabla \mathcal{S} g)^{*}\right\|_{L^{p}(\partial \Omega)} \leq C\|g\|_{L^{p}\left(\partial \Omega, \Lambda^{1} T M\right)}
$$

and if $P$ is a first order differential operator on sections of $\Lambda^{1} T M$, then at almost every boundary point $x \in \partial \Omega$,

$$
\begin{aligned}
\left.(P \mathcal{S} g)\right|_{\partial \Omega_{ \pm}}(x)= & \mp \frac{1}{2} i \sigma(P ; x, \nu) g_{\tan }(x) \\
& + \text { p.v. } \int_{\partial \Omega}\left\langle\left(P_{x} \otimes I_{y}\right) \Gamma(x, y), g(y)\right\rangle d \sigma(y),
\end{aligned}
$$

for any $g \in L^{p}\left(\partial \Omega, \Lambda^{1} T M\right)$. 
(2) For $1<p<\infty$,

$$
\left\|(\mathcal{Q} g)^{*}\right\|_{L^{p}(\partial \Omega)} \leq C\|g\|_{L^{p}\left(\partial \Omega, \Lambda^{1} T M\right)},
$$

uniformly for $g \in L^{p}\left(\partial \Omega, \Lambda^{1} T M\right)$, and

$$
\left.\mathcal{Q} g\right|_{\partial \Omega_{ \pm}}=\mp \frac{1}{2}\langle\nu, g\rangle+\text { p.v. } \int_{\partial \Omega}\langle\Theta(x, y), g(y)\rangle d \sigma(y), \quad x \in \partial \Omega,
$$

for all $g \in L^{p}\left(\partial \Omega, \Lambda^{1} T M\right)$. Also,

$$
L \mathcal{S} g+d \mathcal{Q} g=0 \quad \text { in } \quad \Omega_{ \pm}, \quad \forall g \in L_{-1}^{p}\left(\partial \Omega, \Lambda^{1} T M\right) .
$$

(3) For $0<s<1 / p<1$, the operators

$$
\begin{aligned}
& \mathcal{Q}: B_{-s}^{p, p}\left(\partial \Omega, \Lambda^{1} T M\right) \rightarrow B_{-s+1 / p}^{p, p}\left(\Omega, \Lambda^{1} T M\right) \cap L_{-s+1 / p}^{p}\left(\Omega, \Lambda^{1} T M\right) \\
& \mathcal{Q}: L^{p}\left(\partial \Omega, \Lambda^{1} T M\right) \rightarrow B_{1 / p}^{p, p^{*}}\left(\Omega, \Lambda^{1} T M\right)
\end{aligned}
$$

are well-defined and bounded. Hereafter, $p^{*}:=\max \{p, 2\}$.

(4) For $1 \leq p \leq \infty$ and $0<s<1$, and each $i \geq 0$,

$$
\left\|\operatorname{dist}(\cdot, \partial \Omega)^{s+i-1 / p}\left|\nabla^{i} \mathcal{Q} g\right|\right\|_{L^{p}(\Omega)} \leq C\|g\|_{B_{-s}^{p, p}\left(\partial \Omega, \Lambda^{1} T M\right)}
$$

and

$$
\left\|\operatorname{dist}(\cdot, \partial \Omega)^{1 / 2}|\mathcal{Q} g|\right\|_{L^{2}(\Omega)} \leq C\|g\|_{L_{-1}^{2}\left(\partial \Omega, \Lambda^{1} T M\right)} .
$$

(5) For $1<p<\infty$ and $0 \leq s \leq 1$,

$$
\mathcal{S}: L_{-s}^{p}\left(\partial \Omega, \Lambda^{1} T M\right) \rightarrow B_{-s+1+1 / p}^{p, p^{*}}\left(\Omega, \Lambda^{1} T M\right)
$$

is well-defined and bounded. Moreover, for $1 \leq p \leq \infty$ and $0<s<1$,

$$
\mathcal{S}: B_{-s}^{p, p}\left(\partial \Omega, \Lambda^{1} T M\right) \rightarrow B_{-s+1+1 / p}^{p, p}\left(\Omega, \Lambda^{1} T M\right)
$$

is well-defined and bounded. Finally, if $1<p<\infty$ and $0<s<1$,

$$
\mathcal{S}: B_{-s}^{p, p}\left(\partial \Omega, \Lambda^{1} T M\right) \rightarrow L_{-s+1+1 / p}^{p}\left(\Omega, \Lambda^{1} T M\right)
$$

is well-defined and bounded. Also,

$$
\delta(\mathcal{S} g)=0 \quad \text { in } \quad \Omega_{ \pm}, \quad \forall g \in L_{-1}^{p}\left(\partial \Omega, \Lambda^{1} T M\right)
$$


(6) For $1<p<\infty$,

$$
\left\|(\mathcal{S} g)^{*}\right\|_{L^{p}(\partial \Omega)} \leq C\|g\|_{L_{-1}^{p}\left(\partial \Omega, \Lambda^{1} T M\right)}
$$

and for each $g \in L_{-1}^{p}\left(\partial \Omega, \Lambda^{1} T M\right)$,

$$
\left.(\mathcal{S} g)\right|_{\partial \Omega_{ \pm}}=S g, \quad \text { in } \quad L^{p}\left(\partial \Omega, \Lambda^{1} T M\right) .
$$

Also, if $0 \leq s \leq 1$, then

$$
S: L_{-s}^{p}\left(\partial \Omega, \Lambda^{1} T M\right) \rightarrow L_{1-s, \nu}^{p}(\partial \Omega)
$$

is well-defined and bounded. A similar result is valid on the Besov scale for $0<s<1$ and $1 \leq p \leq \infty$.

(7) For $1 \leq p \leq \infty$ and $0<s<1$, and each $i \geq 0$,

$$
\left\|\operatorname{dist}(\cdot, \partial \Omega)^{s+i-1 / p}\left|\nabla^{i+1} \mathcal{S} g\right|\right\|_{L^{p}(\Omega)} \leq C\|g\|_{B_{-s}^{p, p}\left(\partial \Omega, \Lambda^{1} T M\right)}
$$

and

$$
\left\|\operatorname{dist}(\cdot, \partial \Omega)^{1 / 2}|\nabla \mathcal{S} g|\right\|_{L^{2}(\Omega)} \leq C\|g\|_{L_{-1}^{2}\left(\partial \Omega, \Lambda^{1} T M\right)},
$$

uniformly in $g$.

(8) If $\partial \Omega \in C^{\infty}$ then

$$
S \in O P S^{-1}\left(\partial \Omega ;\left.\Lambda^{1} T M\right|_{\partial \Omega},\left.\Lambda^{1} T M\right|_{\partial \Omega}\right) \quad \text { is strongly elliptic. }
$$

As a prelude to the proof of this theorem, we discuss a couple of auxiliary results of independent interest.

Lemma 3.2 Let $\Omega$ be a Lipschitz domain in $M$ and consider a positive integer $N$. Suppose that the kernel $k(x, y)$ is defined on $M \times M \backslash$ diagonal and satisfies

$$
\left|\nabla_{x}^{i} \nabla_{y}^{j} k(x, y)\right| \leq \kappa \operatorname{dist}(x, y)^{-(n-1-\theta+i+j)}, \quad \forall i=0,1, \ldots, N, \forall j=0,1,
$$

for some non-negative integer $\theta$ and some constant $\kappa>0$ independent of $x, y$. Introduce

$$
\mathcal{K} f(x):=\left\langle\left. k(x, \cdot)\right|_{\partial \Omega}, f\right\rangle, \quad x \in \Omega
$$


where $\langle\cdot, \cdot\rangle$ stands for the natural pairing between classes of distributions and their corresponding test functions on $\partial \Omega$.

Then, for $1 \leq p, p^{\prime} \leq \infty, 1 / p+1 / p^{\prime}=1$, and $0<s<1$, this operator satisfies the estimates

$$
\begin{aligned}
\left\|\operatorname{dist}(\cdot, \partial \Omega)^{s+i-1 / p}\left|\nabla^{\theta+i} \mathcal{K} f\right|\right\|_{L^{p}(\Omega)} & \\
& +\sum_{k=0}^{\theta+i}\left\|\nabla^{[\theta+i-k-1]_{+}} \mathcal{K} f\right\|_{L^{p}(\Omega)} \leq C\|f\|_{\left(B_{s}^{p^{\prime}, p^{\prime}}(\partial \Omega)\right)^{*}}
\end{aligned}
$$

for all $i=0,1, \ldots, N-1$, uniformly for $f$ in $\left(B_{s}^{p^{\prime}, p^{\prime}}(\partial \Omega)\right)^{*}$. Here $[a]_{+}:=\max \{a, 0\}$ and we agree that $\nabla^{0}$ is the identity.

In particular, (3.45) holds in the case when $k(x, y)$ is the Schwartz kernel of a pseudodifferential operator $P \in O P S^{-1-\theta}(M)$. In this later situation, the estimate

$$
\left\|\operatorname{dist}(\cdot, \partial \Omega)^{1 / 2}|\nabla \mathcal{K} f|\right\|_{L^{2}(\Omega)} \leq C\|f\|_{L^{2}(\partial \Omega)}
$$

is also valid provided $\theta=0$ and $\sigma(P ; \xi)$ is odd in $\xi$.

Proof. Consider first (3.45). When $\theta=1$, the cases $p=1$ and $p=\infty$ have been proved in [13]. The range $1<p<\infty$ then follows by Stein's interpolation theorem for analytic families of operators. The extension to other values of $\theta$ follows the same pattern. We omit the details. Finally, (3.46) has been proved in [11].

Proposition 3.3 Let $k(x, y)$ denote the Schwartz kernel of a pseudodifferential operator $P \in O P S^{-1-\theta}(M)$ and recall the integral operator $\mathcal{K}$ associated with $k(x, y)$ as in (3.44). Then, if $\theta=1$,

$$
0<s<1, \quad 1 \leq p \leq \infty \Rightarrow \mathcal{K}: B_{-s}^{p, p}(\partial \Omega) \rightarrow B_{1-s+1 / p}^{p, p}(\Omega) \text { is bounded }
$$

and

$$
0<s<1, \quad 1<p<\infty \Rightarrow \mathcal{K}: B_{-s}^{p, p}(\partial \Omega) \rightarrow L_{1-s+1 / p}^{p}(\Omega) \text { is bounded. }
$$

Also, with $p^{*}$ denoting $\max \{p, 2\}$ and assuming that the principal symbol $\sigma(P ; x, \xi)$ is even in $\xi$,

$$
1<p<\infty \Longrightarrow \mathcal{K}: L^{p}(\partial \Omega) \rightarrow B_{1+1 / p}^{p, p^{*}}(\Omega) \text { is bounded. }
$$

On the other hand, if $\theta=0$ then

$$
0<s<1 / p<1 \Rightarrow \mathcal{K}: B_{-s}^{p, p}(\partial \Omega) \rightarrow B_{-s+1 / p}^{p, p}(\Omega) \cap L_{-s+1 / p}^{p}(\Omega) \text { is bounded }
$$


and, if $\sigma(P ; x, \xi)$ is odd in $\xi$,

$$
1<p<\infty \Longrightarrow \mathcal{K}: L^{p}(\partial \Omega) \rightarrow B_{1 / p}^{p, p^{*}}(\Omega) \text { is bounded }
$$

Proof. The implications in (3.49) and (3.51) are minor variations of results in [13]. Also, the implication in (3.47) for $0<s<1 / p \leq 1$ follows from the estimate (3.45) in Lemma 3.2 (with $\theta=1$ and $i=1$ ) and the real-variable characterization of the membership to Sobolev-Besov spaces from [8] (cf. also Proposition 4.4 in [13]). The range $0 \leq 1 / p<s<1$ is handled similarly (this time take $i=0$ in (3.45)) and the whole range follows by interpolation (cf., e.g., [1], [8]). Furthermore, the implication (3.48) can be proved analogously.

When the same approach is used to establish (3.50) we get only the range $0<$ $s<1 / p<1$ since, this time, only the option $i=1$ works in (3.45).

We are now prepared to present the

Proof of Theorem 3.1. The points (1) and (2) follow directly from definitions and the theory developed in [11]. Also, (3) is an immediate consequence of Proposition 3.3 while (3.31) is seen from (3.45).

Turning attention to (3.32), let us introduce $\Upsilon:=-G(L \Phi-I) \in O P S^{-2}(M)$ and denote by $\Sigma(x, y)$ its Schwartz kernel. Note that $\sigma(\Upsilon ; x, \xi)$, the principal symbol of $\Upsilon$, is even in $\xi$. Now, at the level of Schwartz kernels, the identity $\delta \Upsilon=\Psi$ translates into $\delta_{x} \Sigma(x, y)=\Theta(x, y)$. In order to continue, we need an auxiliary result which we now describe.

Working in local coordinates, let us introduce the first-order tangential derivative operators $\partial / \partial t_{j k}$ on $\partial \Omega$ by setting

$$
\frac{\partial f}{\partial t_{j k}}:=\left.\left(\nu_{j} \partial_{k} f-\nu_{k} \partial_{j} f\right)\right|_{\partial \Omega} .
$$

Here, each $\partial / \partial t_{j k}: L^{p}(\partial \Omega) \rightarrow L_{-1}^{p}(\partial \Omega)=\left(L_{1}^{p}(\partial \Omega)\right)^{*}, 1 / p+1 / q=1$, is defined in a natural way via duality, making use of the integration by parts formula

$$
\int_{\partial \Omega} \frac{\partial f}{\partial t_{j k}} g d \sigma=\int_{\partial \Omega} f \frac{\partial g}{\partial t_{k j}} d \sigma+\int_{\partial \Omega} f g \omega_{j k} d \sigma,
$$

where $\omega_{j k} \in L^{\infty}(\partial \Omega)$ are independent of $f$ and $g$.

With this notation, we have the characterization

$$
L_{-1}^{p}(\partial \Omega) \equiv\left\{\sum_{j, k} \frac{\partial f_{j k}}{\partial t_{j k}}+f_{0} ; f_{j k}, f_{0} \in L^{p}(\partial \Omega)\right\}
$$

plus equivalence of norms.

Returning to the task of proving (3.32), observe that for each $g \in L^{2}(\partial \Omega), \mathcal{Q} g$ obeys the square-function estimate (3.32) thanks to, e.g., (3.27). Thus, granted (3.54), in order to handle the general case $g \in L_{-1}^{2}(\partial \Omega)$, it suffices to only look at $\mathcal{Q}\left(\partial f / \partial t_{j k}\right)$, 
for $f \in L^{2}\left(\partial \Omega, \Lambda^{1} T M\right)$. In this scenario, integrating by parts (cf. (3.53)), allows one to locally write this in the form

$$
\begin{aligned}
\mathcal{Q}\left(\frac{\partial f}{\partial t_{j k}}\right)(x)= & \delta_{x} \int_{\partial \Omega}\left\{\left\langle\partial_{y_{j}} \Sigma(x, y),\left(\nu_{k} f\right)(y)\right\rangle-\left\langle\partial_{y_{k}} \Sigma(x, y),\left(\nu_{j} f\right)(y)\right\rangle\right\} d \sigma(y) \\
& +\mathcal{Q}\left(f \omega_{j k}\right)(x) .
\end{aligned}
$$

Now, the fact that the integral operators $\mathcal{K}_{j}$ associated with the kernels $\partial_{y_{j}} \Sigma(x, y)$ as in (3.44) satisfy (3.46), in concert with (3.55), translates into the desired quadratic estimate for the operator $\mathcal{Q}$ acting on $L_{-1}^{2}(\partial \Omega)$. This finishes the proof of (4).

As for the operator (3.33), its boundedness for $s=0$ follows directly from Proposition 3.3. Then the entire range follows by complex interpolation as soon as we show that

$$
\mathcal{S}: L_{-1}^{p}\left(\partial \Omega, \Lambda^{1} T M\right) \longrightarrow B_{1 / p}^{p, p^{*}}\left(\Omega, \Lambda^{1} T M\right)
$$

is bounded. To this end, once again it suffices to consider only terms of the form

$$
\mathcal{S}\left(\frac{\partial f}{\partial t_{j k}}\right), \quad \text { for } \quad f \in L^{p}\left(\partial \Omega, \Lambda^{1} T M\right)
$$

which, after integrations by parts, look like

$$
\begin{aligned}
\mathcal{S}\left(\frac{\partial f}{\partial t_{j k}}\right)(x)= & \int_{\partial \Omega}\left\{\left\langle\partial_{y_{j}} \Gamma(x, y),\left(\nu_{k} f\right)(y)\right\rangle-\left\langle\partial_{y_{k}} \Gamma(x, y),\left(\nu_{j} f\right)(y)\right\rangle\right\} d \sigma(y) \\
& +\mathcal{S}\left(f \omega_{j k}\right)(x) .
\end{aligned}
$$

Now, Proposition 3.3 applied to the operators with kernels $\partial_{y_{j}} \Gamma(x, y)$, gives

$$
\begin{aligned}
\|\mathcal{S} g\|_{B_{1 / p}^{p, p^{*}}(\Omega)}= & \left\|\mathcal{S}\left(f_{0}+\sum_{j, k} \frac{\partial f_{j k}}{\partial t_{j k}}\right)\right\|_{B_{1 / p}^{p, p^{*}}(\Omega)} \\
\leq & \left\|\mathcal{S} f_{0}\right\|_{B_{1 / p}^{p, p^{*}}(\Omega)}+\sum_{j, k}\left\|\int_{\partial \Omega}\left\langle\partial_{y_{j}} \Gamma, \nu_{k} f_{j k}\right\rangle\right\|_{B_{1 / p}^{p, p^{*}}(\Omega)} \\
& +\sum_{j, k}\left\|\int_{\partial \Omega}\left\langle\partial_{y_{k}} \Gamma, \nu_{j} f_{j k}\right\rangle\right\|_{B_{1 / p}^{p, p^{*}}(\Omega)}+\sum_{j, k}\left\|\mathcal{S}\left(\omega_{j k} f_{j k}\right)\right\|_{B_{1 / p}^{p, p^{*}}(\Omega)} \\
\leq & C\left\|f_{0}\right\|_{L^{p}\left(\partial \Omega, \Lambda^{1} T M\right)}+C \sum_{j k}\left\|f_{j k}\right\|_{L^{p}\left(\partial \Omega, \Lambda^{1} T M\right)} \leq C\|g\|_{L_{-1}^{p}\left(\partial \Omega, \Lambda^{1} T M\right)} .
\end{aligned}
$$

This justifies the boundedness of (3.56) and finishes the proof of the claim having to do with the operator (3.33). 
The boundedness of the operators (3.34)-(3.35) is, in turn, a direct consequence of Proposition 3.3. Also, the identity (3.36) follows immediately from (3.12).

Going further, (3.37) is proved in a similar fashion to (3.27). Next, the general jump-formulas from [11] applied to the operators in the left side of (3.38) give

$$
\begin{aligned}
\left.\mathcal{S}\left(\frac{\partial f}{\partial t_{j k}}\right)\right|_{\partial \Omega_{ \pm}}= & \mp \frac{1}{2} \nu_{j} \nu_{k} f_{\tan }+\text { p.v. } \int_{\partial \Omega}\left\langle\partial_{y_{j}} \Gamma, \nu_{k} f\right\rangle d \sigma \\
& -\left(\mp \frac{1}{2} \nu_{k} \nu_{j} f_{\tan }+\text { p.v. } \int_{\partial \Omega}\left\langle\partial_{y_{k}} \Gamma, \nu_{j} f\right\rangle d \sigma\right)+S\left(f \omega_{j k}\right) \\
= & \text { p.v. } \int_{\partial \Omega}\left\langle\partial_{y_{j}} \Gamma, \nu_{k} f\right\rangle d \sigma-\text { p.v. } \int_{\partial \Omega}\left\langle\partial_{y_{k}} \Gamma, \nu_{j} f\right\rangle d \sigma+S\left(f \omega_{j k}\right) \\
= & S\left(\frac{\partial f}{\partial t_{j k}}\right)
\end{aligned}
$$

In turn, this readily justifies (3.38). With an eye on (3.39), we first claim that the operator $S: L_{-s}^{p}(\partial \Omega) \rightarrow L_{1-s}^{p}(\partial \Omega)$ is well-defined and bounded for $0 \leq s \leq 1$ and $1<p<\infty$. The fact that this is well-defined and bounded when $s=0$ and $1<p<\infty$ has been established in [11]. Then duality (cf. (3.13)) and complex interpolation give the full range $0 \leq s \leq 1$. Note that the same conclusion is valid on the Besov scale (for $0<s<1$ and $1 \leq p \leq \infty)$ from (3.34) and (3.38). To finish the proof of (6) we only need to observe that, by the Divergence Theorem,

$$
\int_{\partial \Omega}\langle\nu, S g\rangle d \sigma=\iint_{\Omega} \delta \mathcal{S} g d \mathrm{Vol}=0
$$

Moving on to (7), observe that (3.40) is a consequence of (3.45), while (3.41) is proved much as (3.32).

Finally, with (8) in mind, we digress momentarily in order to put matters in the proper perspective. Let $\Omega$ be a smooth domain in $M(\operatorname{dim} M=m)$ and recall that $d \sigma$ stands for the surface measure on $\partial \Omega$. Also, let $\mathcal{E}, \mathcal{F} \rightarrow M$ be two smooth vector bundles and fix $p(x, D) \in O P S^{\ell}(M ; \mathcal{E}, \mathcal{F})$, where $-m<\ell<0$. Under these conditions, it follows that

$$
\left.p(x, D)(f d \sigma)\right|_{\Omega} \in C^{0}(\bar{\Omega}, \mathcal{F}), \quad \forall f \in C^{\infty}\left(\partial \Omega,\left.\mathcal{E}\right|_{\partial \Omega}\right) .
$$

Here $f d \sigma$ is the distribution on $M$ (supported on $\partial \Omega$ ) given by

$$
\langle f d \sigma, \varphi\rangle:=\int_{\partial \Omega}\langle f, \varphi\rangle d \sigma .
$$

In fact, if $K(x, y) \in C^{\infty}(M \times M \backslash \operatorname{diag} ; \mathcal{F} \otimes \mathcal{E}) \cap \mathcal{D}^{\prime}(M \times M ; \mathcal{F} \otimes \mathcal{E})$ is the Schwartz kernel of $p$ then, locally,

$$
|K(x, y)| \leq C|x-y|^{-m+\ell}
$$


and one can check that the boundary trace of $\left.p(x, D)(f \sigma)\right|_{\Omega}$ is given by

$$
\left.p(x, D)(f d \sigma)\right|_{\partial \Omega}(x)=\int_{\partial \Omega}\langle K(x, y), f(y)\rangle d \sigma(y), \quad x \in \partial \Omega .
$$

In order to continue we recall a standard result.

Proposition 3.4 With the notation and assumptions of the previous paragraph (in particular, assuming $\left.\partial \Omega \in C^{\infty}\right)$, denote the mapping

$$
\left.C^{\infty}(\partial \Omega, \mathcal{E}) \in f \mapsto p(\cdot, D)(f d \sigma)\right|_{\partial \Omega} \in C^{\infty}(\partial \Omega, \mathcal{F})
$$

by $\widetilde{p}(x, D)$. Then $\widetilde{p}(x, D) \in O P S^{\ell+1}\left(\partial \Omega ;\left.\mathcal{E}\right|_{\partial \Omega},\left.\mathcal{F}\right|_{\partial \Omega}\right)$ and its principal symbol is given by

$$
\sigma(\widetilde{p} ; x, \xi)=\frac{1}{2 \pi} \int_{-\infty}^{+\infty} \sigma(p ; x, \xi+t \nu) d t
$$

where $\nu$ is the unit conormal to $\partial \Omega, \xi \in T_{x}^{*} \partial \Omega, x \in \partial \Omega$.

With this in hand, it follows from (3.65) that

$$
S=\left.\Phi(x, D)(\cdot d \sigma)\right|_{\partial \Omega} \in O P S^{-1}\left(\partial \Omega ;\left.\Lambda^{1} T M\right|_{\partial \Omega},\left.\Lambda^{1} T M\right|_{\partial \Omega}\right) .
$$

Furthermore, since from (3.18) and (3.67)

$$
\langle\sigma(S ; x, \xi) u, u\rangle \geq c|u|^{2}, \quad c>0,
$$

at each $x \in \partial \Omega$ and for all $\xi \in T_{x}^{*} \partial \Omega,|\xi|=1, u \in \Lambda^{1} T_{x} M$, we may conclude that $\mathcal{S}$ is strongly elliptic.

This completes the proof of (8) and, with it, the proof of Theorem 3.1.

\section{Rellich estimate}

In local coordinates, and with the summation convention understood, the Stokes system (with $u$ regarded as a 1 -differential form $u=u_{j} d x_{j}$ ) reads

$$
\left\{\begin{array}{l}
g^{j k} \partial_{j} \partial_{k} u_{s}=\partial_{s} p+\text { first order terms in } u, \quad \forall s, \\
g^{j k} \partial_{k} u_{j}=\text { zero order terms in } u
\end{array}\right.
$$

In this section, we shall find it useful to work with the vector $v=\left(v^{j}\right)_{j}$ obtained from $\left(u_{j}\right)_{j}$ by raising the indices, i.e.

$$
v^{j}:=g^{j k} u_{k}, \forall j, \text { so that } u_{j}=g_{j k} v^{k}, \forall j .
$$

Then (4.1) becomes 


$$
\left\{\begin{array}{l}
a_{j k}^{s l} \partial_{j} \partial_{k} v^{l}=\partial_{s} p+\text { first order terms in } v, \quad \forall s, \\
\partial_{k} v^{k}=\text { zero order terms in } v
\end{array}\right.
$$

where $a_{j k}^{s l}:=g^{j k} g_{s l}$ is a real tensor which is symmetric, i.e., $a_{j k}^{s l}=a_{k j}^{l s}$, and satisfies an ellipticity condition stronger than the standard Legendre-Hadamard condition, namely

$$
a_{j k}^{s l}(x) \zeta_{j}^{s} \zeta_{k}^{l} \geq C|\zeta|^{2}, \quad \forall \zeta \in T_{x}^{*} M \otimes T_{x} M \equiv \operatorname{Hom}\left(T M \otimes T^{*} M, \mathbb{R}\right),
$$

uniformly in $x$; here $\zeta_{j}^{s}=\zeta\left(\partial_{x_{s}}, d x_{j}\right)$ are the components of the $(1,1)$-tensor $\zeta$. Indeed, if we consider the matrices $G:=\left(g^{j k}\right)_{j k}, E:=\left(g_{s l} \zeta_{r}^{s}\right)_{l r}$, then (4.4) can be written the (equivalent) invariant form Trace $\left(G E G E^{t}\right) \geq C|E|^{2}$. In turn, this is readily checked by working with a basis in which $G=\operatorname{diag}\left(\lambda_{1}, \ldots, \lambda_{m}\right)$, for some $\lambda_{j}>0, j=1,2, \ldots, m$.

As in [14], [11], if $h=\left(h_{j}\right)_{j}$ is an arbitrary $C^{1}$-vector field then

$$
\begin{aligned}
\partial_{l}\left[\left(h_{l} a_{j k}^{\alpha \beta}-h_{j} a_{l k}^{\alpha \beta}-h_{k} a_{j l}^{\alpha \beta}\right) \partial_{j} v^{\alpha} \partial_{k} v^{\beta}\right]= & -2 h_{l} \partial_{l} v^{\alpha}\left(a_{j k}^{\alpha \beta} \partial_{j} \partial_{k} v^{\beta}\right) \\
& +\mathcal{O}\left(|\nabla v|^{2}+|v|^{2}\right) .
\end{aligned}
$$

Note that since $v=\left(v^{j}\right)_{j}$ satisfies (4.3), the first term in the right side of (4.5) is $-2 h_{l} \partial_{l} v^{\alpha} \partial_{\alpha} p+\mathcal{O}\left(|\nabla v|^{2}+|v|^{2}\right)$. With this in mind, (4.5) and the Divergence Theorem yield

$$
\begin{aligned}
-2 \iint_{\Omega} h_{l} \partial_{l} v^{\alpha} \partial_{\alpha} p & +\iint_{\Omega} \mathcal{O}\left(|\nabla v|^{2}+|v|^{2}\right) \\
= & \int_{\partial \Omega}\langle n, h\rangle a_{j k}^{\alpha \beta} \partial_{j} v^{\alpha} \partial_{k} v^{\beta} d \sigma_{0} \\
& -\int_{\partial \Omega}\left(n_{l} h_{j} a_{l k}^{\alpha \beta}+n_{l} h_{k} a_{j l}^{\alpha \beta}\right) \partial_{j} v^{\alpha} \partial_{k} v^{\beta} d \sigma_{0}=: I-I I .
\end{aligned}
$$

Here $n=\left(n_{j}\right)_{j}$ and $d \sigma_{0}$ are, respectively, the Euclidean unit normal and surface measure on $\partial \Omega$. Next, since

$$
\begin{aligned}
n_{l} h_{j} a_{l k}^{\alpha \beta} \partial_{j} & =n_{l} h_{l} a_{j k}^{\alpha \beta} \partial_{j}+\text { tangential derivative } \\
n_{l} h_{k} a_{j l}^{\alpha \beta} \partial_{k} & =n_{l} h_{l} a_{j k}^{\alpha \beta} \partial_{k}+\text { tangential derivative }
\end{aligned}
$$

it follows that

$$
\begin{aligned}
I I & =\int_{\partial \Omega} 2 n_{l} h_{l} a_{j k}^{\alpha \beta} \partial_{j} v^{\alpha} \partial_{k} v^{\beta} d \sigma_{0}+\int_{\partial \Omega} \mathcal{O}\left(\left|\nabla_{\tan } v\right||\nabla v|\right) d \sigma_{0} \\
& =2 I+\int_{\partial \Omega} \mathcal{O}\left(\left|\nabla_{\tan } v \| \nabla v\right|\right) d \sigma_{0} .
\end{aligned}
$$


Substituting this back in (4.6) gives

$$
\begin{aligned}
\int_{\partial \Omega}\langle h, n\rangle a_{j k}^{\alpha \beta} \partial_{j} v^{\alpha} \partial_{k} v^{\beta} d \sigma_{0}= & -2 \iint_{\Omega} h_{l} \partial_{l} v^{\alpha} \partial_{\alpha} p \\
& +\int_{\partial \Omega} \mathcal{O}\left(\left|\nabla_{\tan } v\right||\nabla v|\right) d \sigma_{0}+\iint_{\Omega} \mathcal{O}\left(|\nabla v|^{2}+|v|^{2}\right) .
\end{aligned}
$$

Going further, an integration by parts gives

$$
\begin{aligned}
\iint_{\Omega}\left(h_{l} \partial_{l} v^{\alpha}\right) \partial_{\alpha} p & =-\iint_{\Omega} p \partial_{\alpha}\left(h_{l} \partial_{l} v^{\alpha}\right)+\int_{\partial \Omega} h_{l} \partial_{l} v^{\alpha} n_{\alpha} p d \sigma_{0} \\
& =: I I I+I V .
\end{aligned}
$$

Thanks to $\partial_{\alpha} v^{\alpha}=\mathcal{O}(|v|)$, the integrand in $I I I$ is $\mathcal{O}((|\nabla v|+|v|)|p|)$. Also, since $n_{\alpha} h_{l} \partial_{l}=n_{l} h_{l} \partial_{\alpha}+$ tangential derivative, we infer that

$$
\begin{aligned}
I V & =\int_{\partial \Omega}\langle n, h\rangle \partial_{\alpha} v^{\alpha} p d \sigma_{0}+\int_{\partial \Omega} \mathcal{O}\left(\left|\nabla_{\tan } v\right||p|\right) d \sigma_{0} \\
& =\int_{\partial \Omega} \mathcal{O}\left(\left(\left|\nabla_{\tan } v\right|+|v|\right)|p|\right) d \sigma_{0} .
\end{aligned}
$$

One last ingredient needed here is the pointwise estimate

$$
|\nabla v|^{2} \leq C\left(a_{j k}^{\alpha \beta} \partial_{j} v^{\alpha} \partial_{k} v^{\beta}\right),
$$

obtained by using the strong ellipticity condition (4.4) on the coefficients $a_{j k}^{\alpha \beta}$.

Let us now assume that matters have been arranged so that $\langle h, n\rangle \geq \kappa>0$ on $\partial \Omega$ and that the support of $v$ is small. Using (4.11) and the estimates derived above for $I I I$ and $I V$ back in (4.8), we finally arrive at

$$
\begin{aligned}
\int_{\partial \Omega}|\nabla v|^{2} d \sigma_{0} \leq & C \int_{\partial \Omega}\left|\nabla_{\tan } v\right|^{2} d \sigma_{0}+C \int_{\partial \Omega}|v|^{2} d \sigma_{0}+\varepsilon \int_{\partial \Omega}|p|^{2} d \sigma_{0} \\
& +C \iint_{\Omega}\left(|\nabla v|^{2}+|v|^{2}+|p|^{2}\right) d \mathrm{Vol}
\end{aligned}
$$

where $\varepsilon>0$ is arbitrary and the constant $C$ depends only on $\partial \Omega$ and $\varepsilon$. Observe that the Radon-Nikodym derivative $d \sigma_{0} / d \sigma$ belongs to $L^{\infty}(\partial \Omega)$.

Thus, in terms of the original field $u$ and the original surface measure $d \sigma$, this proves (via a standard partition of unity argument) that when the pair $(u, p)$ solves (4.1) on a Lipschitz domain $\Omega$ and satisfies appropriate non-tangential maximal estimates, then 


$$
\begin{aligned}
\int_{\partial \Omega}|\nabla u|^{2} d \sigma \leq & C\|u\|_{L_{1}^{2}\left(\partial \Omega, \Lambda^{1} T M\right)}^{2}+\varepsilon\|p\|_{L^{2}(\partial \Omega)}^{2} \\
& +C\|u\|_{L_{1}^{2}\left(\Omega, \Lambda^{1} T M\right)}^{2}+C\|p\|_{L^{2}(\Omega)}^{2},
\end{aligned}
$$

where, once again, $\varepsilon>0$ is arbitrary and $C=C(\partial \Omega, \varepsilon)>0$.

\section{Pressure estimate}

In this section we complement the Rellich-type estimate (4.13) with an estimate on the pressure. Consider $u \in C_{\mathrm{loc}}^{2}\left(\Omega, \Lambda^{1} T M\right), p \in C_{\mathrm{loc}}^{1}(\Omega)$ satisfying

$$
\left\{\begin{array}{l}
L u+d p=0 \text { in } \Omega, \\
\delta u=0 \text { in } \Omega, \\
u^{*},(\nabla u)^{*}, p^{*} \in L^{2}(\partial \Omega) .
\end{array}\right.
$$

Since $d p=\Delta u+2 \operatorname{Ric} u=\delta d u+2 \operatorname{Ric} u$ in $\Omega$ it follows that

$$
\Delta p=-\delta d p=-\delta \delta d u-2 \delta \operatorname{Ric} u=-2 \delta \circ \operatorname{Ric} u .
$$

Note that $\delta \circ$ Ric is a first-order differential operator on $M$.

Fix a smooth, non-negative function $V \not \equiv 0$ on $M$ such that $V \equiv 0$ on $\bar{\Omega}$ and let $\Omega_{j} \nearrow \Omega$ be an approximating sequence. Among other things, we assume that there are bi-Lipschitz homeomorphisms $\Lambda_{j}$ mapping $\partial \Omega$ onto $\partial \Omega_{j}$. See [20], [12] for more details. Next, set $\Pi_{j}^{V}, \mathcal{D}_{j}^{V}, \mathcal{S}_{j}^{V}$, for Newtonian, double and single layer potential operators associated with $\Delta-V$ and $\Omega_{j}$. Also, denote by $S_{j}^{V}$ the trace of $\mathcal{S}_{j}^{V}$ on $\partial \Omega_{j}$ and by $K_{j}^{V}$ the principal-value versions of $\mathcal{D}_{j}^{V}$ on $\partial \Omega_{j}$. $\left(\mathcal{S}_{j}^{V}\right.$ and $S_{j}^{V}$ are not to be confused with $\mathcal{S}$ and $S$, defined in (3.20)-(3.21).)

Now, (5.2) and the fact that $p \in C^{1}\left(\bar{\Omega}_{j}\right)$, give that in each $\Omega_{j}$,

$$
p=\mathcal{D}_{j}^{V}\left(\left.p\right|_{\partial \Omega_{j}}\right)-\mathcal{S}_{j}^{V}\left(\left.\frac{\partial p}{\partial \nu_{j}}\right|_{\partial \Omega_{j}}\right)+\Pi_{j}^{V}(\Delta p) .
$$

Going to the boundary and using (5.2) yields

$$
\begin{aligned}
\left(\frac{1}{2} I-K_{j}^{V}\right)\left(\left.p\right|_{\partial \Omega_{j}}\right)= & -S_{j}^{V}\left(\left\langle\nu_{j},\left.(d p)\right|_{\partial \Omega_{j}}\right\rangle\right)+\left.\Pi_{j}^{V}(\Delta p)\right|_{\partial \Omega_{j}} \\
= & -S_{j}^{V}\left(\left\langle\nu_{j},\left.(\delta d u)\right|_{\partial \Omega_{j}}\right\rangle\right)-2 S_{j}^{V}\left(\left\langle\nu_{j},\left.(\operatorname{Ric} u)\right|_{\partial \Omega_{j}}\right\rangle\right) \\
& +\left.2 \Pi_{j}^{V}(\delta \circ \operatorname{Ric} u)\right|_{\partial \Omega_{j}} .
\end{aligned}
$$

In order to continue, we bring in a result from [11]. Specifically, an $\ell$-form $f \in$ $L^{2}\left(\partial \Omega, \Lambda^{\ell} T M\right.$ ), is called tangential (to $\partial \Omega$ ) if $\nu \vee f=0$ on $\partial \Omega$, where $\nu \vee \cdot$ is the 
interior product with $\nu$. Also, $f$ is called normal (to $\partial \Omega$ ) if $\nu \wedge f=0$ on $\partial \Omega$. We set $f_{\tan }:=\nu \vee(\nu \wedge f)$ and $f_{\text {nor }}:=\nu \wedge(\nu \vee f)$ so that $f=f_{\text {tan }}+f_{\text {nor }}$ is the orthogonal decomposition of $f$ into tangential and normal parts. Furthermore, we say that an $L^{2}$-tangential form $f$ has its boundary (exterior) co-derivative in $L^{2}$ if there exists an $(\ell-1)$-form in $L^{p}\left(\partial \Omega, \Lambda^{\ell-1} T M\right)$, which we denote by $\delta_{\partial} f$, so that

$$
\int_{\partial \Omega}\langle d \psi, f\rangle d \sigma=\int_{\partial \Omega}\left\langle\psi, \delta_{\partial} f\right\rangle d \sigma \quad \text { for any } \psi \in C^{1}\left(M, \Lambda^{\ell-1} T M\right) .
$$

Then if $w \in C^{1}\left(\Omega, \Lambda^{\ell} T M\right)$ is such that $w^{*},(\delta w)^{*} \in L^{2}(\partial \Omega)$ and $w, \delta w$ have nontangential boundary traces at almost any point on $\partial \Omega$, it follows that

$$
\delta_{\partial}\left(\left.\nu \vee w\right|_{\partial \Omega}\right)=-\left.\nu \vee(\delta w)\right|_{\partial \Omega}
$$

Returning to the analysis of (5.4), we observe that thanks to (5.5) and (5.6),

$$
\begin{aligned}
S_{j}^{V}\left(\left\langle\nu_{j},\left.(\delta d u)\right|_{\partial \Omega_{j}}\right\rangle\right) & =S_{j}^{V}\left(\left.\nu_{j} \vee(\delta d u)\right|_{\partial \Omega_{j}}\right) \\
& =-S_{j}^{V}\left(\delta_{\partial}\left(\left.\nu_{j} \vee(d u)\right|_{\partial \Omega_{j}}\right)\right) \\
& =-\int_{\partial \Omega_{j}}\left\langle\nu_{j}(y) \wedge d_{y} \Gamma^{V}(\cdot, y), d u(y)\right\rangle d \sigma_{j}(y) \\
& =: T_{j}\left(\left.(d u)\right|_{\partial \Omega_{j}}\right),
\end{aligned}
$$

where the last equality defines the boundary integral operator $T_{j}$.

Let now $K^{V}$ stand for the (principal-value) double layer operator on $\partial \Omega$. Since, for any $F \in C^{\infty}(M)$,

$$
\left(\frac{1}{2} I-K_{j}^{V *}\right)\left(\left.F\right|_{\partial \Omega_{j}}\right) \circ \Lambda_{j} \stackrel{L^{2}(\partial \Omega)}{\longrightarrow}\left(\frac{1}{2} I-K^{V *}\right)\left(\left.F\right|_{\partial \Omega}\right), \text { as } j \rightarrow \infty,
$$

(cf. [20], [12]) a standard duality argument gives that

$$
\left(\frac{1}{2} I-K_{j}^{V}\right)\left(\left.p\right|_{\partial \Omega_{j}}\right) \circ \Lambda_{j} \longrightarrow\left(\frac{1}{2} I-K^{V}\right)\left(\left.p\right|_{\partial \Omega}\right) \text { weakly in } L^{2}(\partial \Omega) .
$$

Consequently, since the operator norm of $T_{j}$ as a mapping on $L^{2}\left(\partial \Omega_{j}\right)$ is bounded uniformly in $j$ (cf. [11]), we have

$$
\begin{aligned}
\|\left(\frac{1}{2} I\right. & \left.-K^{V}\right)\left(\left.p\right|_{\partial \Omega}\right) \|_{L^{2}(\partial \Omega)} \\
& \leq \limsup _{j \rightarrow \infty}\left\|\left(\frac{1}{2} I-K_{j}^{V}\right)\left(\left.p\right|_{\partial \Omega_{j}}\right)\right\|_{L^{2}\left(\partial \Omega_{j}\right)} \\
& \leq \limsup _{j \rightarrow \infty}\left(\left\|\left.(d u)\right|_{\partial \Omega_{j}}\right\|_{L^{2}\left(\partial \Omega_{j}\right)}+\left\|\left.u\right|_{\partial \Omega_{j}}\right\|_{L^{2}\left(\partial \Omega_{j}\right)}+\|\nabla u\|_{L^{2}\left(\Omega_{j}\right)}\right) \\
& \leq C\left(\left\|\left.(\nabla u)\right|_{\partial \Omega}\right\|_{L^{2}(\partial \Omega)}+\left\|\left.u\right|_{\partial \Omega}\right\|_{L^{2}(\partial \Omega)}+\|\nabla u\|_{L^{2}(\Omega)}\right) .
\end{aligned}
$$


Now, from [12], $\frac{1}{2} I-K^{V}$ is a Fredholm operator with index zero on $L^{2}(\partial \Omega)$. In particular, there exists a compact operator on $L^{2}(\partial \Omega)$ such that

$$
\|f\|_{L^{2}(\partial \Omega)} \leq C\left\|\left(\frac{1}{2} I-K^{V}\right) f\right\|_{L^{2}(\partial \Omega)}+\|\operatorname{Comp} f\|,
$$

uniformly for $f \in L^{2}(\partial \Omega)$. Thus we have the following pressure estimate:

$$
\begin{aligned}
\left\|\left.p\right|_{\partial \Omega}\right\|_{L^{2}(\partial \Omega)} \leq & C\left\|\left.(\nabla u)\right|_{\partial \Omega}\right\|_{L^{2}(\partial \Omega)} \\
& +C\|u\|_{L^{2}(\partial \Omega)}+C\|\nabla u\|_{L^{2}(\Omega)}+\left\|\operatorname{Comp}\left(\left.p\right|_{\partial \Omega}\right)\right\| .
\end{aligned}
$$

Parenthetically, let us point out that an $L^{p}$-version of (5.12) also holds, as long as $2-\varepsilon<p<\infty$ for some $\varepsilon=\varepsilon(\Omega)>0$.

\section{$6 \quad$ Invertibility of layer potential operators}

Our goal in this section is to establish invertibility of the operator $S$, defined by (3.21), as a mapping between a variety of Sobolev spaces and Besov spaces. The precise result is given in Theorem 6.1 below.

To begin, take $g \in L^{2}\left(\partial \Omega, \Lambda^{1} T M\right)$, and consider

$$
u:=\mathcal{S} g, \quad p:=\mathcal{Q} g \quad \text { in } M \backslash \partial \Omega,
$$

where $\mathcal{S}$ and $\mathcal{Q}$ are defined in (3.20)-(3.22). In particular, $L^{2}\left(\partial \Omega, \Lambda^{1} T M\right) \ni g \mapsto$ $\left.p\right|_{\partial \Omega} \in L^{2}(\partial \Omega)$ is bounded, so the mapping $g \mapsto \operatorname{Comp}\left(\left.p\right|_{\partial \Omega}\right)$ is compact on $L^{2}(\partial \Omega)$. Also, the Rellich-type estimate (4.13) ensures that

$$
\left\|\left.(\nabla u)\right|_{\partial \Omega}\right\|_{L^{2}(\partial \Omega)} \leq C\left\|\left.u\right|_{\partial \Omega}\right\|_{L_{1}^{2}(\partial \Omega)}+\|\operatorname{Comp}(g)\|+\varepsilon\left\|\left.p\right|_{\partial \Omega}\right\|_{L^{2}(\partial \Omega)}
$$

for $\varepsilon>0$ arbitrary and $C=C(\partial \Omega, \varepsilon)>0$.

At this stage in our analysis, the aim is to bound $S: L^{2}(\partial \Omega) \rightarrow L_{1}^{2}(\partial \Omega)$ from below (modulo compact operators) and (6.2), when used in concert with the jump relations (3.26), is a useful step in this direction. However, due the failure of $\Phi$ to be elliptic, this estimate alone will eventually allow us to control only the tangential part of $g$ in the desired fashion, as is apparent from (3.26). In turn, the normal component of $g$ is retrievable from the jumps of the pressure function (cf. (3.28)), which is the reason for which we have derived the estimates in $\S 5$. For the time being, we note that (5.12) plus (6.2), and their versions for $\Omega_{-}$, yield in the current context

$$
\left\|\left.p\right|_{\partial \Omega_{ \pm}}\right\|_{L^{2}(\partial \Omega)} \leq C\|S g\|_{L_{1}^{2}(\partial \Omega)}+\|\operatorname{Comp}(g)\| .
$$

To continue, let us denote by $\operatorname{Def}(u) \nu$ the 1 -form defined by $X \mapsto \operatorname{Def}(u)(\nu, \tilde{X})$ (recall that tilde stands for the canonical identification between vector fields and 1-forms induced by the metric). Then, directly from (4.13), 


$$
\left\|\left.\operatorname{Def}(u) \nu\right|_{\partial \Omega}\right\|_{L^{2}\left(\partial \Omega_{ \pm}\right)} \leq C\|S g\|_{L_{1}^{2}(\partial \Omega)}+\|\operatorname{Comp}(g)\|+\varepsilon\|g\|_{L^{2}(\partial \Omega)},
$$

where $\varepsilon>0$ is arbitrary and $C=C(\partial \Omega, \varepsilon)>0$.

Recall next that $\left(u \otimes_{S} v\right)(X, Y)=\frac{1}{2}[\langle u, X\rangle\langle v, Y\rangle+\langle u, Y\rangle\langle v, X\rangle]$ is the symmetric product of 1-forms. Then, dropping the dependence of symbols on the point $x \in \partial \Omega$, we have

$$
\begin{aligned}
\left.\operatorname{Def}(u) \nu\right|_{\partial \Omega_{+}}-\left.\operatorname{Def}(u) \nu\right|_{\partial \Omega_{-}} & =-i[\sigma(\operatorname{Def} ; \nu) \sigma(\Phi ; \nu) g](\nu, \cdot) \\
& =\left(\nu \otimes_{S}(\nu \vee(\nu \wedge g))\right)(\nu, \cdot) \\
& =\frac{1}{2} \nu \vee(\nu \wedge g)=\frac{1}{2} g_{\tan } .
\end{aligned}
$$

Here we have used the readily checked fact that if $\omega \in \Lambda^{1} T M$ is tangential to $\partial \Omega$ then $\left(\nu \otimes_{S} \omega\right)(\nu, \cdot)=\frac{1}{2} \omega$. On the other hand,

$$
\left.\nu p\right|_{\partial \Omega_{+}}-\left.\nu p\right|_{\partial \Omega_{-}}=-\nu \wedge(\nu \vee g)=-g_{\text {nor }}
$$

Hence we obtain

$$
g=\left.(2 \operatorname{Def}(u) \nu-p \nu)\right|_{\partial \Omega_{+}}-\left.(2 \operatorname{Def}(u) \nu-p \nu)\right|_{\partial \Omega_{-}} .
$$

In turn, this and (6.3)-(6.4) yield the important estimate

$$
\|g\|_{L^{2}(\partial \Omega)} \leq C\|S g\|_{L_{1}^{2}(\partial \Omega)}+\|\operatorname{Comp}(g)\|, \quad \forall g \in L^{2}\left(\partial \Omega, \Lambda^{1} T M\right) .
$$

Suppose next that $u, p$ solve the homogeneous version of (5.1), i.e.,

$$
L u+d p=0, \quad \delta u=0,\left.\quad u\right|_{\partial \Omega}=0, \quad p^{*}, u^{*},(\nabla u)^{*} \in L^{2}(\partial \Omega) .
$$

Then, successive integrations by parts give

$$
\begin{aligned}
2 \iint_{\Omega}|\operatorname{Def} u|^{2} d \mathrm{Vol} & =\iint_{\Omega}\langle u, L u\rangle d \mathrm{Vol}=\iint_{\Omega}\langle u, d p\rangle d \mathrm{Vol} \\
& =\int_{\partial \Omega}\langle u, \nu \wedge p\rangle d \sigma+\iint_{\Omega}\langle\delta u, p\rangle d \mathrm{Vol}=0 .
\end{aligned}
$$

Hence the hypotheses in (6.9) imply $\operatorname{Def} u=0$ on $\Omega$, but

$$
\operatorname{Def} u=0 \text { on } \Omega,\left.\quad u\right|_{\partial \Omega}=0 \Longrightarrow u=0 \text { on } \Omega \text { and } p=c_{+}=\text {const. on } \Omega .
$$

In fact, the hypothesis $(\nabla u)^{*} \in L^{2}(\partial \Omega)$ plus those in (6.11) imply $u \in L_{1,0}^{2}(\Omega)$ (the closure of $C_{0}^{\infty}(\Omega)$ in $L_{1}^{2}(\Omega)$ ). Then $u$ extended by 0 on $M \backslash \Omega$, gives $\tilde{u}$ satisfying Def $\tilde{u}=$ 
0. Hence $\tilde{u} \in C^{\infty}(M)$ generates a group of isometries of $M$. The hypothesis (3.1) gives $\tilde{u}=0$ on $M$. Alternatively, since this group fixes each point in the nonempty open set $M \backslash \bar{\Omega}$, the group is readily seen to be trivial. As another alternative, we have $L \tilde{u}=0$ on $M$, and since $L$ is a second order elliptic operator with scalar principal symbol, unique continuation gives $\tilde{u}=0$ on $M$.

We are prepared to describe the kernel of $S$ on $L^{2}\left(\partial \Omega, \Lambda^{1} T M\right)$. Consider $g \in$ $L^{2}\left(\partial \Omega, \Lambda^{1} T M\right)$ such that $S g=0$ and set $u:=\mathcal{S} g, p:=\mathcal{Q} g$ in $\Omega_{ \pm}$. Since $\left.u\right|_{\partial \Omega_{ \pm}}=0$, the reasoning above implies $u \equiv 0$ and $p=c_{ \pm}=$const. in $\Omega_{ \pm}$. In particular, from (6.7) we see that $g \in \operatorname{span}(\nu)=\mathbb{R} \nu$. Conversely, if $g=c \nu, c \in \mathbb{R}$, then, by the Divergence Theorem,

$$
S g(x)=c \int_{\partial \Omega}\langle\Gamma(x, y), \nu(y)\rangle d \sigma(y)=c \iint_{\Omega} \delta_{y} \Gamma(x, y) d \operatorname{Vol}_{y}=0 .
$$

Hence,

$$
\operatorname{Ker}\left(S: L^{2}\left(\partial \Omega, \Lambda^{1} T M\right) \rightarrow L^{2}\left(\partial \Omega, \Lambda^{1} T M\right)\right)=\mathbb{R} \nu .
$$

We are now ready to state the main result of this section.

Theorem 6.1 There exists $\varepsilon=\varepsilon(\Omega)>0$ such that for each $2-\varepsilon<p<2+\varepsilon$ and $0 \leq s \leq 1$,

$$
S: L_{-s}^{p}\left(\partial \Omega, \Lambda^{1} T M\right) / \mathbb{R} \nu \rightarrow L_{1-s}^{p}\left(\partial \Omega, \Lambda^{1} T M\right) / \mathbb{R} \nu \text { is invertible. }
$$

Furthermore, similar results are valid on the Besov scale for $p$ near 2 and $0<s<1$.

Proof. Most of the main ingredients are in place already. First, (6.13) and the estimate (6.8) imply that, for any Lipschitz domain $\Omega$,

$$
S: L_{\nu}^{2}(\partial \Omega) \rightarrow L_{1, \nu}^{2}(\partial \Omega) \text { is injective with closed range. }
$$

Recall, from (3.13), that $S^{*}=S$ and notice that

$$
\left(L_{\nu}^{2}(\partial \Omega)\right)^{*} \cong L^{2}\left(\partial \Omega, \Lambda^{1} T M\right) / \mathbb{R} \nu, \quad\left(L_{1, \nu}^{2}(\partial \Omega)\right)^{*} \cong L_{-1}^{2}\left(\partial \Omega, \Lambda^{1} T M\right) / \mathbb{R} \nu
$$

Next, on smooth domains, we claim that

$$
S: L_{-1}^{2}\left(\partial \Omega, \Lambda^{1} T M\right) / \mathbb{R} \nu \rightarrow L^{2}\left(\partial \Omega, \Lambda^{1} T M\right) / \mathbb{R} \nu \text { is injective. }
$$

Indeed, in this setting, as a corollary of the ellipticity of $S$ (cf. (8) in Theorem 3.1) the kernel of this operator is contained in $L^{2}\left(\partial \Omega, \Lambda^{1} T M\right) / \mathbb{R} \nu$. Then the conclusion follows from (6.13). Using (6.15) in concert with the dual of (6.17) leads to the conclusion

$$
\partial \Omega \in C^{\infty} \Longrightarrow S: L_{\nu}^{2}(\partial \Omega) \rightarrow L_{1, \nu}^{2}(\partial \Omega) \text { is invertible. }
$$


Now, the extra smoothness assumption on $\partial \Omega$ may be eliminated, i.e., we may return to the usual assumption that $\partial \Omega$ is only Lipschitz continuous. This may be done by a limiting argument which utilizes (6.15), (6.18) and the fact that the constant $C$ in (6.8) depends only on the Lipschitz character of $\Omega$. More specifically, fix a field $X \in C^{\infty}(M)$ such that $\int_{\partial \Omega}\langle X, \nu\rangle d \sigma=1$ and an approximating sequence $\Omega_{j} \nearrow \Omega$, $\partial \Omega_{j} \in C^{\infty}$. Also, to a fixed, arbitrary smooth field $F$ on $M$ associate the sequence

$$
f_{j}:=\left.F\right|_{\partial \Omega_{j}}-\left.\lambda_{j} X\right|_{\partial \Omega_{j}}, \text { where } \lambda_{j}:=\frac{\int_{\partial \Omega_{j}}\left\langle F, \nu_{j}\right\rangle d \sigma_{j}}{\int_{\partial \Omega_{j}}\left\langle X, \nu_{j}\right\rangle d \sigma_{j}} .
$$

Note that $f_{j} \in L_{1, \nu_{j}}^{2}\left(\partial \Omega_{j}\right)$ and, under the natural identification of $\partial \Omega_{j}$ with $\partial \Omega$, it converges to $\left.F\right|_{\partial \Omega}-\left.\lambda X\right|_{\partial \Omega}$, where $\lambda:=\int_{\partial \Omega}\langle F, \nu\rangle d \sigma$. If $S_{j}$ is the Stokes single layer on $\partial \Omega_{j}$, set $g_{j}:=S_{j}^{-1} f_{j} \in L_{\nu_{j}}^{2}\left(\partial \Omega_{j}\right)$. Now, a semi-standard argument based on (6.8) gives that there exists a finite, positive constant $C_{0}$, independent of $j$, so that

$$
\|f\|_{L^{2}\left(\partial \Omega_{j}\right)} \leq C_{0}\left\|S_{j} f\right\|_{L_{1}^{2}\left(\partial \Omega_{j}\right)}, \quad \forall f \in L_{\nu_{j}}^{2}\left(\partial \Omega_{j}\right) ;
$$

see, e.g., Lemma 7.3 in [12] for details in similar circumstances. In particular, from this we see that $\left(g_{j}\right)_{j}$ is a bounded sequence in $L^{2}$, so we can assume that it converges weakly to some $g$. Then, for any smooth field $G$ on $M$,

$$
\begin{aligned}
\int_{\partial \Omega}\left\langle S g,\left.G\right|_{\partial \Omega}\right\rangle d \sigma & =\int_{\partial \Omega}\left\langle g, S\left(\left.G\right|_{\partial \Omega}\right)\right\rangle d \sigma=\lim _{j \rightarrow \infty} \int_{\partial \Omega_{j}}\left\langle g_{j}, S_{j}\left(\left.G\right|_{\partial \Omega_{j}}\right)\right\rangle d \sigma_{j} \\
& =\lim _{j \rightarrow \infty} \int_{\partial \Omega_{j}}\left\langle f_{j},\left.G\right|_{\partial \Omega_{j}}\right\rangle d \sigma_{j}=\int_{\partial \Omega}\left\langle\left. F\right|_{\partial \Omega}-\left.\lambda X\right|_{\partial \Omega},\left.G\right|_{\partial \Omega}\right\rangle d \sigma .
\end{aligned}
$$

Thus, $S g=\left.F\right|_{\partial \Omega}-\left.\lambda X\right|_{\partial \Omega}$ so that

$$
\left\{\left.F\right|_{\partial \Omega} ; F \in C^{\infty}\left(M, \Lambda^{1} T M\right), \int_{\partial \Omega}\langle F, \nu\rangle d \sigma=0\right\} \subseteq \operatorname{Range}(S),
$$

for any Lipschitz domain $\Omega$. Hence $S: L_{\nu}^{2}(\partial \Omega) \rightarrow L_{1, \nu}^{2}(\partial \Omega)$ has dense range and, ultimately, is invertible thanks to (6.15).

This proves the desired conclusion about (6.14) when $p=2$ and $s=1$. In fact, duality and interpolation allow us to cover the case $0 \leq s \leq 1$. The extension to $2-\varepsilon<p<2+\varepsilon$ is achieved from what we have proved so far by invoking well-known stability results, given, e.g., in [16] or [9]. Finally, the result can be transferred to the Besov scale via real interpolation.

In closing, let us point out that for $2-\varepsilon<p<2+\varepsilon$ and $0 \leq s \leq 1$,

$$
S: L_{-s}^{p}\left(\partial \Omega, \Lambda^{1} T M\right) \rightarrow L_{1-s}^{p}\left(\partial \Omega, \Lambda^{1} T M\right) \text { is Fredholm with index zero. }
$$

In fact, $\operatorname{dim} \operatorname{Ker} S=\operatorname{dim}$ Coker $S=1$. 


\section{Boundary problems for the Stokes system}

Retaining our basic notation and assumptions from the previous section, we now study the Dirichlet problem for the Stokes system. Our analysis here extends the main results in [6], obtained in the flat, Euclidean setting.

Theorem 7.1 Let $\Omega$ be an arbitrary Lipschitz domain in $M$. For $1<p<\infty$ consider the boundary value problem

$$
(B V P)\left\{\begin{array}{l}
u \in C_{\mathrm{loc}}^{2}\left(\Omega, \Lambda^{1} T M\right), \quad p \in C_{\mathrm{loc}}^{1}(\Omega), \\
L u+d p=0 \quad \text { in } \Omega, \\
\delta u=0 \quad \text { in } \Omega, \\
u^{*} \in L^{p}(\partial \Omega), \\
\left.u\right|_{\partial \Omega}=f \in L_{\nu}^{p}(\partial \Omega) .
\end{array}\right.
$$

Then there exists $\varepsilon=\varepsilon(\Omega)>0$ such that for all $p \in(2-\varepsilon, 2+\varepsilon)$ the following hold:

(1) $(B V P)$ is solvable uniquely for $u$, and uniquely modulo constants for $p$. Moreover,

$$
\left\|u^{*}\right\|_{L^{p}(\partial \Omega)} \leq C\|f\|_{L^{p}\left(\partial \Omega, \Lambda^{1} T M\right)} .
$$

(2) The following regularity statement is valid:

$$
(\nabla u)^{*} \in L^{p}(\partial \Omega) \Longleftrightarrow f \in L_{1, \nu}^{p}(\partial \Omega) .
$$

Moreover, if $f \in L_{1, \nu}^{p}(\partial \Omega)$ then $p^{*} \in L^{p}(\partial \Omega)$ and

$$
\left\|p^{*}\right\|_{L^{p}(\partial \Omega)}+\left\|(\nabla u)^{*}\right\|_{L^{p}(\partial \Omega)}+\left\|u^{*}\right\|_{L^{p}(\partial \Omega)} \leq C\|f\|_{L_{1}^{p}\left(\partial \Omega, \Lambda^{1} T M\right)} .
$$

(3) For $0 \leq s \leq 1$, we have

$$
f \in L_{s, \nu}^{p}(\partial \Omega) \Longrightarrow u \in B_{s+1 / p}^{p, p^{*}}\left(\Omega, \Lambda^{1} T M\right),
$$

plus a natural estimate (recall that $\left.p^{*}=\max \{p, 2\}\right)$.

(4) On the scale of Besov spaces (retaining the necessary compatibility condition $\left.\int_{\partial \Omega}\langle f, \nu\rangle d \sigma=0\right)$, we have:

$$
\begin{aligned}
& \text { if } 0<s<1, f \in B_{s}^{p, p}(\partial \Omega) \Rightarrow u \in B_{s+1 / p}^{p, p}(\Omega) \cap L_{s+1 / p}^{p}(\Omega), \\
& \text { if } 1-1 / p<s<1, f \in B_{s}^{p, p}(\partial \Omega) \Rightarrow p \in B_{s+1 / p-1}^{p, p}(\Omega) \cap L_{s+1 / p-1}^{p}(\Omega),
\end{aligned}
$$

plus natural estimates. 
Proof. Existence is provided in the form

$$
u:=\mathcal{S}\left(S^{-1} f\right), \quad p:=\mathcal{Q}\left(S^{-1} f\right) \quad \text { in } \Omega .
$$

By Theorem 6.1 this is meaningful as long as $2-\varepsilon<p<2+\varepsilon$. Also, (7.6) and Theorem 3.1 ensure that the solution just constructed has all the desired properties.

There remains uniqueness. For a fixed $x_{0} \in \Omega$, we construct a Green function $G\left(x, x_{0}\right)$, a double form of type $(1,1)$, with pole at $x_{0}$. Specifically, for $x \in \Omega$ we set

$$
G\left(x, x_{0}\right):=\Gamma\left(x, x_{0}\right)-(\mathcal{S} \bar{\otimes} \mathrm{I})\left[\left(S^{-1} \otimes \mathrm{I}\right)\left(\left.\Gamma\left(\cdot, x_{0}\right)\right|_{\partial \Omega}\right)\right](x) .
$$

Note that this is well-defined since $\left.\Gamma\left(\cdot, x_{0}\right)\right|_{\partial \Omega} \in L_{1, \nu}^{q}(\partial \Omega) \bar{\otimes} \Lambda^{1} T_{x_{0}} M$, where $1 / p+1 / q=$ 1. Indeed,

$$
\int_{\partial \Omega}\left\langle\Gamma\left(x, x_{0}\right), \nu(x)\right\rangle d \sigma(x)=-\iint_{\Omega_{-}} \delta_{x} \Gamma\left(x, x_{0}\right) d \operatorname{Vol}_{x}=0
$$

as $\delta_{x} \Gamma\left(x, x_{0}\right)=0$ in $\Omega_{-}$. Next, let $\Omega_{j} \nearrow \Omega$ be an approximating sequence as before and denote by $G_{j}\left(x, x_{0}\right)$ the Green function corresponding to $\Omega_{j}$. If $1 / p+1 / q=1$, given what we have proved so far, we have:

$$
\left\{\begin{array}{l}
L_{x} G_{j}\left(x, x_{0}\right)+d_{x} \Theta\left(x, x_{0}\right)=\operatorname{Dirac}_{x_{0}}(x) \text { in } \Omega_{j}, \\
\delta_{x} G_{j}\left(x, x_{0}\right)=0 \text { in } \Omega_{j}, \\
\left.G_{j}\left(\cdot, x_{0}\right)\right|_{\partial \Omega_{j}}=0 \\
\sup _{j}\left\{\left\|\left(G_{j}\left(\cdot, x_{0}\right)\right)^{*}\right\|_{L^{q}\left(\partial \Omega_{j}\right)}+\left\|\left(\nabla_{x} G_{j}\left(\cdot, x_{0}\right)\right)^{*}\right\|_{L^{q}\left(\partial \Omega_{j}\right)}\right\} \leq C<+\infty .
\end{array}\right.
$$

Granted these, we may write

$$
u\left(x_{0}\right)=\iint_{\Omega_{j}}\left\langle L_{x} G_{j}\left(x, x_{0}\right)+d_{x} \Theta\left(x, x_{0}\right), u(x)\right\rangle d \mathrm{Vol}_{x} .
$$

Integrating by parts in $d_{x}$ leads only to a boundary term since $\delta u=0$. By (7.9) and Hölder's inequality, the latter is controlled in terms of $\left\|\left.u\right|_{\partial \Omega_{j}}\right\|_{L^{p}(\partial \Omega)}$. For the remaining part, involving $L G_{j}$, we also integrate by parts. The resulting solid integral will contain $L u=d p$. After a new integration by parts in $d$, this becomes

$$
\begin{aligned}
\iint_{\Omega_{j}}\left\langle G_{j}\left(x, x_{0}\right), d p(x)\right\rangle d \mathrm{Vol}_{x}= & \iint_{\Omega_{j}}\left\langle\delta_{x} G_{j}\left(x, x_{0}\right), p(x)\right\rangle d \mathrm{Vol}_{x} \\
& +\int_{\partial \Omega_{j}}\left\langle G_{j}\left(x, x_{0}\right), \nu(x)\right\rangle p(x) d \sigma(x)=0
\end{aligned}
$$

since $\delta_{x} G_{j}\left(x, x_{0}\right)=0$ and $\left.G_{j}\left(\cdot, x_{0}\right)\right|_{\partial \Omega_{j}}=0$.

Returning to the main line of discussion, the boundary integrals arising in the process of integrating by parts in $\iint_{\Omega_{j}}\left\langle L G_{j}, u\right\rangle d$ Vol can be dominated in absolute 
value by $\int_{\partial \Omega_{j}}|u|\left|\nabla G_{j}\right| d \sigma$, since $\left.G_{j}\left(\cdot, x_{0}\right)\right|_{\partial \Omega_{j}}=0$. In turn, this is further controlled by $C\left\|\left.u\right|_{\partial \Omega_{j}}\right\|_{L^{p}\left(\partial \Omega_{j}\right)}$, since $\left\|\left(\nabla G_{j}\left(\cdot, x_{0}\right)\right)^{*}\right\|_{L^{p}\left(\partial \Omega_{j}\right)} \leq C$, uniformly in $j$.

In summary we have obtained a Poisson-type integral representation formula from which the estimate

$$
\left|u\left(x_{0}\right)\right| \leq C\left(x_{0}, p, \partial \Omega\right)\left\|\left.u\right|_{\partial \Omega_{j}}\right\|_{L^{p}\left(\partial \Omega_{j}\right)}
$$

can be easily derived via Hölder's inequality. Letting $j \rightarrow \infty$ yields $u\left(x_{0}\right)=0$. Since $x_{0}$ is arbitrary, it follows that $u \equiv 0$ in $\Omega$. Thus, $d p=0$, so that $p$ is constant.

Corollary 7.2 Assume that $L u+d p=0, \delta u=0$ in $\Omega$ and that $u^{*} \in L^{2}(\partial \Omega)$. Then the square-function estimates

$$
\begin{gathered}
\iint_{\Omega}|\nabla u(x)|^{2} \operatorname{dist}(x, \partial \Omega) d \operatorname{Vol}_{x} \leq C \int_{\partial \Omega}|u|^{2} d \sigma \\
\iint_{\Omega}\left|\nabla^{i} p(x)\right|^{2} \operatorname{dist}(x, \partial \Omega)^{2 i+1} d \operatorname{Vol}_{x} \leq C_{i} \int_{\partial \Omega}|u|^{2} d \sigma, \quad i=0,1, \ldots,
\end{gathered}
$$

are valid for some $C, C_{i}>0$ depending exclusively on $\Omega$ and $i$. When $i=0, p$ in (7.14) is considered modulo additive constants.

Furthermore, if $p$ is sufficiently close to 2 and $\left.u\right|_{\partial \Omega} \in B_{1-s}^{p, p}\left(\partial \Omega, \Lambda^{1} T M\right)$ for some $s \in(0,1)$ then, for each $i \geq 0$,

$$
\left\|\operatorname{dist}(\cdot, \partial \Omega)^{s+i-1 / p}\left|\nabla^{i} p\right|\right\|_{L^{p}(\Omega)} \leq C_{i}\|u\|_{B_{1-s}^{p, p}\left(\partial \Omega, \Lambda^{1} T M\right)}
$$

(with the same convention as above when $i=0$ ) and

$$
\left\|\operatorname{dist}(\cdot, \partial \Omega)^{s+i-1 / p}\left|\nabla^{i+1} u\right|\right\|_{L^{p}(\Omega)} \leq C_{i}\|u\|_{B_{1-s}^{p, p}\left(\partial \Omega, \Lambda^{1} T M\right)} .
$$

Finally, if in addition, $(\nabla u)^{*} \in L^{2}(\partial \Omega)$, then

$$
\iint_{\Omega}\left|\nabla^{2} u(x)\right|^{2} \operatorname{dist}(x, \partial \Omega) d \operatorname{Vol}_{x} \leq C \int_{\partial \Omega}\left(\left|\nabla_{\tan } u\right|^{2}+|u|^{2}\right) d \sigma
$$

and, for each $i=0,1, \ldots$,

$$
\iint_{\Omega}\left|\nabla^{i+1} p(x)\right|^{2} \operatorname{dist}(x, \partial \Omega)^{2 i+1} d \operatorname{Vol}_{x} \leq C_{i} \int_{\partial \Omega}\left(\left|\nabla_{\tan } u\right|^{2}+|u|^{2}\right) d \sigma .
$$

Proof. The estimates (7.13), (7.15), (7.16), (7.17), as well as (7.14) and (7.18) with $i=0$ are consequences of the integral representation of the solution of the Stokes boundary problem in Theorem 6.1 and the mapping properties of the layer potentials involved. The extension to $i \geq 1$ for (7.14) and (7.18) then follows from the case $i=0$ and interior estimates (cf., e.g., Proposition 3.4 in [13]).

It should be pointed out that in the flat, Euclidean setting, results similar to the ones presented in Corollary 7.2 have been obtained in [6] and [3], via a different approach. 


\section{The Stokes operator}

In this section we define the Stokes operator and study its properties. To do this, we introduce the spaces

$$
\begin{aligned}
& W^{0}(\Omega):=\left\{w \in L^{2}\left(\Omega, \Lambda^{1} T M\right) ; \delta w=0,\langle\nu, w\rangle=0\right\}, \\
& W^{1}(\Omega):=\left\{w \in L_{1}^{2}\left(\Omega, \Lambda^{1} T M\right) ; \delta w=0,\left.w\right|_{\partial \Omega}=0\right\} .
\end{aligned}
$$

We define the unbounded operator $\mathcal{D}: W^{0}(\Omega) \rightarrow L^{2}\left(\Omega, S^{2} T \Omega\right)$ by setting

$$
\operatorname{Dom}(\mathcal{D}):=W^{1}(\Omega) \quad \text { and } \quad \mathcal{D}:=\left.\operatorname{Def}\right|_{W^{1}(\Omega)} .
$$

The Stokes operator $A$ is then defined as

$$
A:=2 \mathcal{D}^{*} \mathcal{D} \text {. }
$$

Proposition 8.1 The operator $\mathcal{D}$ is a closed, densely defined operator. Hence $A$ is a positive self-adjoint operator.

Proof. That $W^{1}(\Omega)$ is dense in $W^{0}(\Omega)$ is essentially well known; cf. Lemma 5.1 in Chapter 17 of [18]. The setting there was that of smoothly bounded domains, but the proof works as well in the present context, given that

$$
W^{0}(\Omega)^{\perp}=\left\{d p ; p \in L_{1}^{2}(\Omega)\right\}
$$

remains valid for Lipschitz domains. That $\mathcal{D}$ is closed follows from Gårding's inequality, which applies since the operator Def has an injective symbol. The self-adjointness of $A$ then follows by von Neumann's theorem.

Standard functional analysis then gives $\operatorname{Dom}\left(A^{1 / 2}\right)=\operatorname{Dom}(\mathcal{D})=W^{1}(\Omega)$ and we set

$$
W^{s}(\Omega):=\operatorname{Dom}\left(A^{s / 2}\right), \quad s \geq 0,
$$

in agreement with the (already defined) cases $s=0$ and $s=1$. We want to know more about the spaces $W^{s}(\Omega)$, particularly their embedding into the standard Sobolev and Besov spaces. When $\partial \Omega$ is smooth one has $W^{s}(\Omega) \subset L_{s}^{2}(\Omega)$ for $0 \leq s \leq 2$, as was shown by Solonnikov. One cannot expect such a result when $\Omega$ is a general Lipschitz domain, but there are nontrivial and useful results to be obtained. We start with the following lemma.

Lemma 8.2 For any $u \in \operatorname{Dom}(A)$ there exist a unique $f \in W^{0}(\Omega)$ and a unique, modulo additive constants, $p \in L^{2}(\Omega)$ such that

$$
L u+d p=f \quad \text { in } \quad \Omega
$$

In fact, $f=A u$. 
Proof. Essentially, this is well-known. We include a proof here mostly to make sure that our analytical assumptions suffice. The uniqueness issue is handled much like in (6.10)-(6.11). As for existence, note that

$$
\begin{aligned}
& u \in \operatorname{Dom}(A) \Longleftrightarrow u \in W^{1}(\Omega) \text { and } \exists f \in W^{0}(\Omega) \\
& \text { such that } \iint_{\Omega}\langle f, v\rangle=\iint_{\Omega}\langle\operatorname{Def} u, \operatorname{Def} v\rangle, \quad \forall v \in W^{1}(\Omega) .
\end{aligned}
$$

In particular, $\omega:=L u-f \in L_{-1}^{2}\left(\Omega, \Lambda^{1} T M\right)$ satisfies

$$
\iint_{\Omega}\langle\omega, v\rangle d \mathrm{Vol}=0, \quad \forall v \in W^{1}(\Omega) .
$$

Our goal is to show that the above implies $\omega=d p$ for some $p \in L^{2}(\Omega)$. For starters, with $E^{\circ}$ denoting the annihilator of $E$, we note that (8.8) simply says that

$$
\omega \in\left[\operatorname{Ker}\left(\delta: L_{1,0}^{2}\left(\Omega, \Lambda^{1} T M\right) \rightarrow L^{2}(\Omega)\right)\right]^{\circ},
$$

where $L_{1,0}^{2}(\Omega)$ denotes the closure of $C_{0}^{\infty}(\Omega)$ in $L_{1}^{2}(\Omega)$. Since $\delta$ above is the adjoint of

$$
d: L^{2}(\Omega) \longrightarrow L_{-1}^{2}\left(\Omega, \Lambda^{1} T M\right)
$$

and since all the spaces involved are reflexive, we may conclude that the annihilator in (8.9) coincides with the closure of the image of the operator (8.10) (cf. Theorems 4.7 and 4.12 in [15]). Thus, in order to finish the proof we only need to show that (8.10) has closed range.

When $\Omega$ is an Euclidean domain this is essentially well-known (see Lemme 7.1, p. 187 in [14]; it can be also proved via duality and a result of Bogovski [2]-cf. (8.13) below). Via pull-back, we can therefore transport it to Lipschitz domains on manifolds which are contained in coordinate charts. Nonetheless, this local version of the result we are seeking self-improves via a patching argument. More specifically, let $\Omega \subset M$ be arbitrary and consider $\left(D_{j}\right)_{j}$ a finite open cover of $\Omega$ with sufficiently small Lipschitz domains.

For each $j$, the operator $d: L^{2}\left(D_{j}\right) \longrightarrow L_{-1}^{2}\left(D_{j}, \Lambda^{1} T M\right)$ has, from what we know so far, closed range and finite dimensional kernel. Thus, there exists a compact operator $\mathrm{Comp}_{j}$ on $L^{2}\left(D_{j}\right)$ and $C_{j}>0$ so that

$$
\|g\|_{L^{2}\left(D_{j}\right)} \leq C_{j}\|d g\|_{L_{-1}^{2}\left(D_{j}, \Lambda^{1} T M\right)}+\left\|\operatorname{Comp}_{j}(g)\right\|, \quad \forall g \in L^{2}\left(D_{j}\right)
$$

If we take $\left(\psi_{j}\right)_{j}$ to be a finite, smooth partition of unity subordinated to $\left(D_{j}\right)_{j}$ then, for any $g \in L^{2}(\Omega)$,

$$
\|g\|_{L^{2}(\Omega)} \leq \sum_{j}\left\|\psi_{j} g\right\|_{L^{2}\left(D_{j}\right)}
$$




$$
\begin{aligned}
& \leq C \sum_{j}\left\|d\left(\psi_{j} g\right)\right\|_{L_{-1}^{2}\left(D_{j}, \Lambda^{1} T M\right)}+\left\|\operatorname{Comp}_{j}(g)\right\| \\
& \leq C\|d g\|_{L_{-1}^{2}\left(\Omega, \Lambda^{1} T M\right)}+\|\operatorname{Comp}(g)\|
\end{aligned}
$$

for some $C>0$ and some compact operator on $L^{2}(\Omega)$. Now (8.12) clearly implies that the operator (8.10) has closed range, as desired.

Remark. Parenthetically, we would like to point out that as a byproduct of the ( $L^{p}$-version of the) above argument, we obtain an extension of a theorem from [2] to the effect that

$$
\delta: L_{1,0}^{p}\left(\Omega, \Lambda^{1} T M\right) \rightarrow\left\{f \in L^{p}(\Omega) ; \iint_{\Omega} f=0\right\} \text { is onto for each } 1<p<\infty .
$$

Indeed, this readily follows from what we have proved so far plus the easily checked fact that $\delta\left[C_{0}^{\infty}\left(\Omega, \Lambda^{1} T M\right)\right]$ is dense in $\left\{f \in L^{p}(\Omega) ; \iint_{\Omega} f=0\right\}$.

Returning to the context of (8.6), from the integral identity (6.10), also valid in the present situation, it follows that $(u, p)$ is the unique solution of the problem

$$
\left\{\begin{array}{l}
u \in L_{1}^{2}\left(\Omega, \Lambda^{1} T M\right), p \in L^{2}(\Omega), \\
L u+d p=f \in L^{2}\left(\Omega, \Lambda^{1} T M\right), \\
\delta u=0 \text { in } \Omega, u=0 \text { on } \partial \Omega .
\end{array}\right.
$$

In order to derive finer global estimates for $u$, we extend $f$ to $\tilde{f} \in L^{2}\left(M, \Lambda^{1} T M\right)$ by assigning it the value zero on $M \backslash \Omega$. We obtain a solution $(v, q)$ of $(8.14)$ with $\Omega$ replaced by $M$ and $f$ by $\tilde{f}$. In this case we have

$$
v \in L_{2}^{2}\left(M, \Lambda^{1} T M\right), \quad q \in L_{1}^{2}(M) .
$$

We now derive regularity results for $u$ by writing $u, p$ in the form

$$
u=\left.v\right|_{\Omega}-w, \quad p=\left.q\right|_{\Omega}-r
$$

where $(w, r)$ solves

$$
\left\{\begin{array}{l}
w \in C_{\mathrm{loc}}^{2}\left(\Omega, \Lambda^{1} T M\right), r \in C_{\mathrm{loc}}^{1}(\Omega), \\
L w+d r=0 \text { in } \Omega, \\
\delta w=0 \text { in } \Omega, \\
\left.w\right|_{\partial \Omega}=\operatorname{Tr} v \text { on } \partial \Omega, \\
w^{*},(\nabla w)^{*}, r^{*} \in L^{p}(\partial \Omega) .
\end{array}\right.
$$

For this program to work, we need to verify several things. First, observe that $\int_{\partial \Omega}\langle\operatorname{Tr} v, \nu\rangle d \sigma=0$ by the Divergence Theorem, since $\delta v=0$ in $\Omega$. Second, we note that 


$$
\left.\nabla v \in L_{1}^{2}(M) \Rightarrow \nabla v\right|_{\partial \Omega} \in L_{1 / 2}^{2}(\partial \Omega) \subset L^{p}(\partial \Omega), \quad \forall p \leq \frac{2 m}{m-2},
$$

if $\operatorname{dim} M=m \geq 3$ (with the obvious modification when $m=2$ ). In concert with (7.4)-(7.5), this also implies that $w \in L_{1}^{2}\left(\Omega, \Lambda^{1} T M\right)$ and $r \in L^{2}(\Omega)$.

With all these preliminaries taken care of, and granted the validity of all the constraints we have encountered so far, it follows from (8.16) and the regularity theory developed in Theorem 7.1 that $w \in B_{1+1 / p}^{p, p^{*}}(\Omega)$. This gives

$$
u \in L_{2}^{2}(\Omega)+B_{1+1 / p}^{p, p^{*}}(\Omega), \quad \forall p \in(2-\varepsilon, 2+\varepsilon) \cap\left(1, \frac{2 m}{m-2}\right) .
$$

Well known embedding theorems then yield the following result, which extends Theorem 2.17 of [3], established there in the flat-space, three-dimensional Euclidean setting.

Theorem 8.3 In any dimension $m$, there exists $p=p(\Omega)>2 m /(m-1)$ such that

$$
\operatorname{Dom}(A) \subseteq L_{1}^{p}(\Omega) \cap L_{3 / 2}^{2}(\Omega) .
$$

Consequently when $\operatorname{dim} \Omega=3$, we have

$$
\operatorname{Dom}(A) \subseteq C^{\alpha}(\bar{\Omega})
$$

for some $\alpha>0$.

\section{The initial value problem for the Navier-Stokes equations}

In this section we shall adapt the semigroup approach of Fujita-Kato [7] for the NavierStokes initial-value problem to the setting of Lipschitz subdomains in Riemannian manifolds. Our argument will follow parallel to that carried out in a narrower context in $[19]$.

Let us recall the general set-up. For $\Omega \subset M$, Lipschitz domain, and the 1-forms $f$, $u_{0}$, playing the roles of the external force and initial velocity, the initial value problem for the Navier-Stokes equations with "no-slip" boundary condition reads

$$
(I B V P)\left\{\begin{array}{l}
\frac{\partial u}{\partial t}+\nu L u+\nabla_{u} u+d p=f \text { in } \mathbb{R}^{+} \times \Omega \\
\delta u=0 \text { in } \mathbb{R}^{+} \times \Omega \\
\left.u(\cdot, t)\right|_{\partial \Omega}=0 \text { for any } t \\
\left.u(\cdot, 0)\right|_{\partial \Omega}=u_{0} \text { in } \Omega .
\end{array}\right.
$$


Here $\nabla$ is the covariant derivative, and $\nu$ is a positive constant, the so-called "viscosity." Let us point out that, as is well known, for divergence-free vector fields $u$ there holds $\nabla_{u} u=-\operatorname{Def}^{*}(u \otimes u)$.

Recall the spaces $W^{s}(\Omega)$ defined in the previous section. If $u_{0} \in W^{1}(\Omega)$, the sense in which the first PDE in (9.1) is to be understood is by demanding that

$$
\begin{aligned}
\frac{d}{d t}\langle u, v\rangle_{L^{2}(\Omega)} & +\left\langle\nabla_{u} u, v\right\rangle_{L^{2}(\Omega)} \\
& +2 \nu\langle\operatorname{Def} u, \operatorname{Def} v\rangle_{L^{2}(\Omega)}=\langle f, v\rangle_{L^{2}(\Omega)}, \quad \forall v \in W^{1}(\Omega) .
\end{aligned}
$$

In order to extend this interpretation to other types of initial data, denote by $P$ the Leray projection, i.e., the orthogonal projection of $L^{2}\left(\Omega, \Lambda^{1} T M\right)$ onto the (closed) subspace $W^{0}(\Omega)$. This allows one to recast (9.2) in the form

$$
\frac{\partial u}{\partial t}+\nu A u+P\left(\nabla_{u} u\right)=P f, \quad u(0)=u_{0}
$$

where $A=P L$ is the Stokes operator, defined in the previous section. Using a standard device, we construct a local solution to the initial value problem by converting (9.3) into an integral equation:

$$
u(t)=e^{-t \nu A} u_{0}-\int_{0}^{t} e^{(s-t) \nu A} P\left[\operatorname{Def}^{*}(u(s) \otimes u(s))+f(s)\right] d s=\Psi u(t) .
$$

We want to find a fixed point of $\Psi$ on $C\left(I, W^{s}(\Omega)\right)$, for $I=\left[0, t_{0}\right]$ with $T_{0}>0$ and some $s>0$, to be described more precisely below. For the standard contraction mapping argument to work, we want to pick a Banach space $Y$ such that

$$
\Phi: W^{s}(\Omega) \rightarrow Y \text { is Lipschitz, uniformly on bounded sets, }
$$

where $\Phi(u):=P \operatorname{Def}^{*}(u \otimes u)$, and such that, for some $b<1$,

$$
\left\|e^{-t A} g\right\|_{W^{s}(\Omega)} \leq C t^{-b}\|g\|_{Y},
$$

for $t \in(0,1]$. Also assume $P f(t)$ is continuous in $t$ with values in $Y$. It is straightforward to verify that $\Psi$ is a contraction on $C\left(I, W^{s}(\Omega)\right)$ for $T_{0}$ sufficiently small, when these conditions are met.

To proceed, we take $Y:=W^{0}(\Omega)$. The condition (9.6) holds by standard functional analysis provided $s<2$ if we choose $b:=s / 2$. Indeed (9.6) is then a consequence of the fact that $(t A)^{s / 2} e^{-t A}$ is uniformly bounded on $W^{i}(\Omega), i=0,1$, for $t \in[0,1]$, and this follows from the Spectral Theorem (here it is also useful to recall that $A^{-s / 2}$ is bounded and invertible from $W^{0}(\Omega)$ to $\left.W^{s}(\Omega)\right)$. As for $(9.5)$, it will hold provided

$$
M: W^{s}(\Omega) \rightarrow L_{1}^{2}(\Omega, T M \otimes T M), \quad M(u):=u \otimes u,
$$


is well-defined and bounded. Since $W^{1}(\Omega) \subset L_{1}^{2}(\Omega, T M)$, we see that the desired conclusion about $(9.7)$ holds provided $s \in[1,2)$ and

$$
W^{s}(\Omega) \subset L^{\infty}(\Omega) .
$$

By Theorem 8.3, if $\operatorname{dim} \Omega=3$ we have

$$
\operatorname{Dom}(A) \subset L_{1}^{p}(\Omega), \quad \text { for some } p>m=3 .
$$

In such a case we have

$$
\begin{aligned}
W^{s}(\Omega)=\operatorname{Dom}\left(A^{s / 2}\right) & =\left[W^{0}(\Omega), \operatorname{Dom}(A)\right]_{s / 2} \\
& \hookrightarrow\left[L^{2}(\Omega), L_{1}^{p}(\Omega)\right]_{s / 2} \\
& \hookrightarrow L_{s / 2}^{q(s)}(\Omega)
\end{aligned}
$$

with

$$
q(s)=\frac{2}{1-s(1 / 2-1 / p)} .
$$

A sufficient condition for $L_{s / 2}^{q(s)}(\Omega)$ to embed in $L^{\infty}(\Omega)$ is

$$
\frac{q(s) s}{2}>m, \quad \text { i.e., } \quad \frac{2}{1+2(1 / m-1 / p)}<s<2 .
$$

Hence we have the following.

Theorem 9.1 Let $\Omega \subset M$ be an arbitrary Lipschitz domain and assume that $\operatorname{dim} M=$ $m \leq 3$. Then there exists $s_{0}=s_{0}(\partial \Omega) \in(1,2)$ such that (9.8) holds for any $s \in\left(s_{0}, 2\right)$.

As a consequence, given any such $s \in\left(s_{0}, 2\right)$, there exists $T_{0}>0$ such that for any $u_{0} \in W^{s}(\Omega), f \in C^{0}\left(\mathbb{R}^{+}, L^{2}\left(\Omega, \Lambda^{1} T M\right)\right)$, the initial boundary problem (9.1) has a unique solution $u \in C^{0}\left(\left[0, T_{0}\right], W^{s}(\Omega)\right)$.

\section{References}

[1] J. Bergh and J. Löfström, Interpolation spaces. An introduction, Springer-Verlag, 1976.

[2] M.E. Bogovski, Solution of some vector analysis problems connected with operators div and grad, Trudy Seminar N. L. Sobolev, No. 1, 80, Akademia Nauk SSSR, Sibirskoe Otdelenie Matematiki, Nowosibirsk, (1980), 5-40.

[3] R. M. Brown and Z. Shen, Estimates for the Stokes operator in Lipschitz domains, Indiana Univ. Math. J., 44 (1995), 1183-1206. 
[4] P. Deuring and W. von Wahl, Strong solutions of the Navier-Stokes system in Lipschitz bounded domains, Math. Nach., 171 (1995), 111-148.

[5] D. Ebin and J. Marsden, Groups of diffeomorphisms and the motion of an incompressible fluid, Annals of Math., 92 (1970), 102-163.

[6] E. Fabes, C. Kenig, and G. Verchota, The Dirichlet problem for the Stokes system on Lipschitz domains, Duke Math. J., 57 (1988), 769-793.

[7] H. Fujita and T. Kato, On the Navier-Stokes initial value problem. I, Archive for Rat. Mech. Anal., Vol. 16, No. 4 (1964), 269-315.

[8] D. Jerison and C. Kenig, The inhomogeneous Dirichlet problem in Lipschitz domains, J. Funct. Anal., 130 (1995), 161-219.

[9] N. Kalton and M. Mitrea, Stability results on interpolation scales of quasi-Banach spaces and applications, Trans. Amer. Math. Soc. 350 (1998), 3903-3922.

[10] O. A. Ladyzhenskaya, The Mathematical Theory of Viscous Incompressible Flow, Gordon \& Breach, New York, 1969.

[11] D. Mitrea, M. Mitrea and M. Taylor, Layer potentials, the Hodge Laplacian and global boundary problems in non-smooth Riemannian manifolds, Mem. Amer. Math. Soc., Vol. 150 No. 713, 2001.

[12] M. Mitrea and M. Taylor, Boundary layer methods for Lipschitz domains in Riemannian manifolds, J. Funct. Anal. 163 (1999), 181-251.

[13] M. Mitrea and M. Taylor, Potential theory on Lipschitz domains in Riemannian manifolds: Sobolev-Besov space results and the Poisson problem, J. Funct. Anal., Vol. 176 No. 1 (2000), 1-79.

[14] J. Nečas, Les Méthodes Directes en Théorie des Équations Élliptiques, Academia, Prague, 1967.

[15] W. Rudin, Functional Analysis, 2nd ed., Int. Series in Pure and Appl. Math., McGrawHill, New York, 1991.

[16] I. Sneiberg, Spectral properties of linear operators in interpolation families of Banach spaces, Mat. Issled. 9 (1974), 214-229.

[17] V. A. Solonnikov, On estimates of the tensor Green's function for some BVP's, Dokl. Akad. Nauk SSSR, 130 (1960), 988-991.

[18] M. Taylor, Partial Differential Equations, Vol. I-III, Springer-Verlag, 1996.

[19] M. Taylor, Incompressible fluid flows on rough domains, in the Proceedings of the International Conference on Semigroups and Applications, California, (1999).

[20] G. Verchota, Layer potentials and boundary value problems for Laplace's equation in Lipschitz domains, J. Funct. Anal., 59 (1984), 572-611. 
Marius Mitrea

Department of Mathematics

University of Missouri at Columbia

Columbia, MO 65211, USA

Michael Taylor

Department of Mathematics

University of North Carolina at Chapel Hill

Chapel Hill, NC 27599, USA 